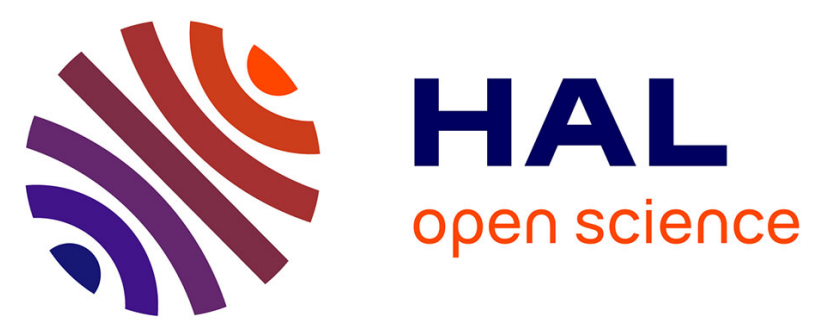

\title{
Characterization of Histone Deacetylase 8 (HDAC8) Selective Inhibition Reveals Specific Active Site Structural and Functional Determinants
}

Martin Marek, Tajith Shaik, Tino Heimburg, Alokta Chakrabarti, Julien Lancelot, Elizabeth Ramos-Morales, Cyrielle da Veiga, Dmitrii Kalinin, Jelena Melesina, Dina Robaa, et al.

\section{To cite this version:}

Martin Marek, Tajith Shaik, Tino Heimburg, Alokta Chakrabarti, Julien Lancelot, et al.. Characterization of Histone Deacetylase 8 (HDAC8) Selective Inhibition Reveals Specific Active Site Structural and Functional Determinants. Journal of Medicinal Chemistry, 2018, 61 (22), pp.10000-10016. 10.1021/acs.jmedchem.8b01087 . hal-02118693

\section{HAL Id: hal-02118693 \\ https://cnrs.hal.science/hal-02118693}

Submitted on 29 Dec 2021

HAL is a multi-disciplinary open access archive for the deposit and dissemination of scientific research documents, whether they are published or not. The documents may come from teaching and research institutions in France or abroad, or from public or private research centers.
L'archive ouverte pluridisciplinaire HAL, est destinée au dépôt et à la diffusion de documents scientifiques de niveau recherche, publiés ou non, émanant des établissements d'enseignement et de recherche français ou étrangers, des laboratoires publics ou privés. 


\section{Characterization of histone deacetylase 8 (HDAC8) selective inhibition}

\section{reveals specific active site structural and functional determinants}

Martin Marek ${ }^{1 \dagger \neq}$, Tajith B. Shaik ${ }^{1 \ddagger}$, Tino Heimburg ${ }^{2}$, Alokta Chakrabarti ${ }^{3}$, Julien Lancelot ${ }^{4}$, Elizabeth Ramos-Morales $^{1}$, Cyrielle Da Veiga ${ }^{5}$, Dmitrii Kalinin ${ }^{6}$, Jelena Melesina ${ }^{2}$, Dina Robaa ${ }^{2}$, Karin Schmidtkunz ${ }^{3}$, Takayoshi Suzuki ${ }^{7}$, Ralph Holl ${ }^{8}$, Eric Ennifar ${ }^{5}$, Raymond J. Pierce ${ }^{4}$, Manfred Jung ${ }^{3}$, Wolfgang Sippl ${ }^{2} \&$ Christophe Romier ${ }^{1 *}$

${ }^{1}$ Institut de Génétique et Biologie Moléculaire et Cellulaire (IGBMC), Département de Biologie Structurale Intégrative, Université de Strasbourg, Centre National de la Recherche Scientifique (CNRS UMR7104), Institut National de la Santé et de la Recherche Médicale (INSERM U1258), 1 rue Laurent Fries, 67404 Illkirch Cedex, France.

${ }^{2}$ Institute of Pharmacy, Martin-Luther-Universität Halle-Wittenberg, Wolfgang-Langenbeck-Straße 4, 06120 Halle/Saale, Germany.

${ }^{3}$ Institute of Pharmaceutical Sciences, Albert-Ludwigs-Universität Freiburg, Albertstraße 25, 79104 Freiburg, Germany.

${ }^{4}$ Université de Lille, CNRS, Inserm, CHU Lille, Institut Pasteur de Lille, U1019 - UMR 8204- CIIL Centre d'Infection et d'Immunité de Lille, F-59000 Lille, France.

${ }^{5}$ Architecture et Réactivité de l’ARN, Institut de Biologie Moléculaire et Cellulaire (IBMC), UPR 9004 du CNRS, Université de Strasbourg, 15 Rue René Descartes, 67084 Strasbourg Cedex, France.

${ }^{6}$ Institute of Pharmaceutical and Medicinal Chemistry, University of Münster, Corrensstr. 48, 48149 Münster, Germany. 
${ }^{7}$ Graduate School of Medical Science, Kyoto Prefectural University of Medicine, 1-5 ShimogamohangiCho, Sakyo-Ku, 606-0823 Kyoto, Japan - CREST, Japan Science and Technology Agency (JST), 4-18 Honcho Kawaguchi, 332-0012 Saitama, Japan.

${ }^{8}$ Institute of Organic Chemistry, Department of Chemistry, University of Hamburg, Martin-Luther-KingPlatz 6, 20146 Hamburg, Germany.

ABSTRACT: Metal-dependent histone deacetylases (HDACs) are key epigenetic regulators that represent promising therapeutic targets for the treatment of numerous human diseases. Yet, the currently FDA-approved HDAC inhibitors non-specifically target at least several of the eleven structurally similar but functionally different HDAC isozymes, which hampers their broad usage in clinical settings. Selective inhibitors targeting single HDAC isozymes are being developed, but precise understanding in molecular terms of their selectivity remains sparse. Here, we show that HDAC8-selective inhibitors adopt a L-shaped conformation required for their binding to a HDAC8-specific pocket formed by HDAC8 catalytic tyrosine and HDAC8 L1 and L6 loops. In other HDAC isozymes, a L1-L6 lock sterically prevents L-shaped inhibitor binding. Shielding of the HDAC8-specific pocket by protein engineering decreases potency of HDAC8-selective inhibitors and affects catalytic activity. Collectively, our results unravel key HDAC8 active site structural and functional determinants important for the design of nextgeneration chemical probes and epigenetic drugs. 


\section{INTRODUCTION}

Acetylation of lysine residues in proteins is a major signaling mark that impacts most cellular processes ${ }^{1-}$

${ }^{3}$. In the cell nucleus, acetylation of histones has been shown to be essential for modulating chromatin structure and for acting in epigenetic signaling that drives and regulates nuclear mechanisms, cellular processes and development ${ }^{1,3,4}$. Protein lysine acetylation is a reversible process relying on the opposing effects of acetyltransferases and deacetylases ${ }^{3,5,6}$. In addition, the acetylation marks on lysines are recognized by epigenetic readers harboring structural modules (e.g. bromodomains) that enable the recruitment of cellular effectors to specific subcellular and genomic loci ${ }^{5,7}$.

Due to the functional importance of acetylation mechanisms, their deregulation has been linked with multiple human diseases, including cancer ${ }^{4,8-11}$. The reversibility of acetylation and the possibility of modulating recognition of acetylated lysines by bromodomains provide a way to pharmacologically modulate acetylation pathways. Thus, epigenetic regulators involved in these pathways represent important therapeutic targets 4 , 8-10, 12, 13 .

Accordingly, among the currently approved epigenetic drugs, a majority (Vorinostat (SAHA), Romidepsin, Belinostat, Panobinostat and Chidamide) target lysine deacetylases ${ }^{13-15}$. The family of lysine deacetylases has been divided into four classes depending on their folds and their sequence similarities. Classes I, II (IIa and IIb), and IV adopt an arginase-deacetylase $\alpha / \beta$ fold and rely on a zinc ion for activity (thereafter referred to as histone deacetylases or HDACs) ${ }^{6}$. Class III deacetylases are referred to as sirtuins and adopt a Rossmann fold, relying on NAD ${ }^{+}$for activity ${ }^{6}$ Eleven HDACs and seven sirtuins are found in humans.

The currently approved drugs against lysine deacetylases target only proteins from the HDAC family. However, these drugs show poor selectivity against single members of the structurally similar but 
functionally different human HDAC isozymes, targeting at least two and generally more than two HDAC isozymes, thus hampering their broad therapeutic usage ${ }^{13,15}$. Several small-molecule inhibitors exhibiting selectivity for specific HDACs have been developed. PCI-34051 and NCC-149 were among the first HDAC isozyme-selective inhibitors discovered ${ }^{16-18}$. These two aromatic hydroxamate derivatives show high selectivity for human HDAC8 (hHDAC8), an HDAC isozyme that has been shown to be overexpressed in several cancers ${ }^{19-21}$ and whose mutations can lead to the Cornelia de Lange syndrome ${ }^{22-}$ 24.

Specifically, PCI-34051, which is an indole-based derivative, is currently among the most selective HDAC8 inhibitors with a selectivity index of 290 and 400 for HDAC6 and HDAC1, respectively, making it a strong chemical tool for studying the biological role of HDAC8 in vivo ${ }^{16,25-28}$. In addition, our work on HDAC8 from the human-pathogenic flatworm Schistosoma mansoni (smHDAC8) has led to the design of new selective HDAC8 inhibitors targeting both the human and schistosome enzymes or, for some of them, showing selectivity towards schistosome HDAC $8^{29,30}$.

To date, the experimental structural and mechanistic bases underlying HDAC8 selective inhibition by PCI-34051, NCC-149 and other related selective compounds remain poorly understood. Structural work on HDAC8 and computational studies have suggested that selective inhibitors target specific structural features of HDAC8 active site, potentially making use of the malleability of this active site ${ }^{17,18,31-34}$. Yet, no clear experimental evidence has been provided so far. To address this issue, we have dissected the molecular basis of HDAC8-selective inhibition by combining biochemical, biophysical and crystallographic studies on hHDAC8 and smHDAC8.

Our results reveal that HDAC8-selective inhibitors bind into a specific HDAC8-selective pocket formed by the active site catalytic tyrosine and by residues from the L1 and L6 loops. This specific enzymeligand recognition is favored by the constrained L-shaped conformation of HDAC8-selective inhibitors. 
This selective binding relies on a specific conformation of the HDAC8 L6 loop and a shorter L1 loop that are not observed in any other HDAC isozyme. Our mutational studies further reveal HDAC8 structural determinants that support HDAC8 selective inhibition and function. Collectively, our results highlight the structural/functional similarities and dissimilarities between the various HDAC isozymes and pave the way for the development of new HDAC isozyme-selective chemical probes for cell biological research and inhibitors to treat human diseases.

\section{RESULTS}

\section{In vitro and in vivo effects of PCI-34051 and NCC-149}

PCI-34051 and NCC-149 have been developed to target human HDAC8 (hHDAC8) selectively ${ }^{16-18}$. To investigate whether these inhibitors also target Schistosoma mansoni HDAC8 (smHDAC8), we have looked at their inhibition and binding to smHDAC8. As a comparison, we have used the highly potent but non-selective HDAC inhibitor Quisinostat (QSN) which is in Phase II of clinical trials ${ }^{35,36 .}$

Measurements of the maximal-half inhibitory concentration $\left(\mathrm{IC}_{50}\right)$ showed that all three inhibitors possess activity in the submicromolar range against hHDAC8 and smHDAC8. NCC-149 showed the most potent inhibition, followed by QSN and PCI-34051 (Figure 1). Measurement of the thermodynamic parameters using Isothermal Titration Calorimetry (ITC) confirmed the inhibition results obtained, the equilibrium dissociation constant $(\mathrm{Kd})$ values determined being in the same range as the corresponding IC $_{50}$ values, with the exception of QSN that showed a lower Kd value for smHDAC8 (Figures 1 and 2).

The biological effects, especially the anti-cancer properties of PCI-34051 and NCC-149 in various cell types have been characterized ${ }^{16-18,25-28}$, and we have previously shown that pan-HDAC inhibitors affect schistosome pathogens $^{30,37}$. We therefore asked whether PCI-34051 and NCC-149 could also have antiparasitic effect on schistosomes. Our various biological assays confirmed that PCI-34051 and NCC-149 
affect the pathogens, triggering their apoptosis (Figure S1). These results demonstrate that both hHDAC8 and smHDAC8 can be used for studying HDAC8 inhibition by PCI-34051 and NCC-149.

So far, few structures of HDACs in complex with selective inhibitors have been solved. Moreover, in many HDAC/inhibitor structures, the active site of the HDAC and the bound inhibitor are involved in extensive crystal packing contacts. This complicates the delineation between biologically-relevant and crystal packing-driven conformations and interactions of the HDAC active site loops and the inhibitors. Therefore, in addition to the co-crystallization attempts of hHDAC8 with inhibitors, we have used the possibility offered by our apo smHDAC8 crystals to look at HDAC8/inhibitor interactions in a crystal lattice-open environment ${ }^{30}$.

Despite intensive efforts, we were not able to obtain well-diffracting crystals of hHDAC8 in complex with PCI-34051, NCC-149 and QSN. In contrast, soaking experiments of apo smHDAC8 crystals with all three inhibitors were successful and yielded high resolution complex structures (Figure 3; Table S1).

\section{Binding mode of QSN to smHDAC8}

Analysis of the smHDAC8/QSN structure revealed that QSN adopts a straight conformation as its piperidine-pyrimidine linker allows limited conformational flexibility (Figure 3A). The QSN hydroxamate warhead coordinates the catalytic zinc and simultaneously interacts via hydrogen bonding with the histidine dyad, H141 and H142 (hHDAC8 H142 and H143) and with the catalytic tyrosine Y341 (hHDAC8 Y306) hydroxyl, as commonly observed for most other hydroxamate-containing HDAC inhibitors. Furthermore, the QSN piperidine-pyrimidine linker is sandwiched between the side chains of smHDAC8 F151 (hHDAC8 F152) and F216 (hHDAC8 F208), where it forms planar $\pi$ - $\pi$ stacking and non-polar contacts. 
Specifically, the QSN piperidine ring adopts a chair conformation, which allows the QSN methyl-aminomethyl linker to form a hydrogen bond (2.4 $\AA$ ) with the carboxyl group of smHDAC8 D100 (hHDAC8 D101), a conserved class I HDAC residue that has been shown to interact with the backbone of incoming acetylated peptides ${ }^{33}{ }^{38}$. Finally, the QSN capping methyl-indole group is solvent exposed, making minimal non-polar contacts with Y99 (hHDAC8 Y100). Interestingly, we previously observed a very similar binding mode to smHDAC8 for another pan-HDAC inhibitor, M344, including an interaction between D100 and the M344 internal amide group ${ }^{30}$. The M344 conformation is less constrained by its linker, which suggests that this binding mode is common to and favored by many pan-HDAC inhibitors. In agreement, a similar binding mode was also observed upon SAHA binding to human HDAC2 in a crystal lattice-free environment ${ }^{39}$.

\section{Binding mode of PCI-34051 to smHDAC8}

The PCI-34051 hydroxamate warhead interacts with the catalytic zinc and active site residues as observed for QSN (Figure 3B). However, in contrast to QSN, the hinge connecting the central indolebased spacer and the methoxyphenyl group of PCI-34051 favours binding of its capping group onto the side chain of smHDAC8 Y341 (hHDAC8 Y306). This tyrosine, together with the catalytic zinc, has been shown in hHDAC8 to be involved in catalysis by polarizing the leaving acetyl group of the incoming acetylated lysine ${ }^{33}$. Here, the methoxyphenyl capping group is perpendicularly $\left(86^{\circ}\right)$ oriented over the aromatic ring of this tyrosine, which favours T-shaped $\pi-\pi$ stacking (4.9 $\AA$ ). Thus, the binding of PCI34051 onto Y341 is favored by the L-shape of this inhibitor.

The methoxyphenyl capping group of PCI-34051 is positioned in close vicinity to the smHDAC8 L6 loop, being inserted in a small pocket shaped by the side chains of P291 and H292 (hHDAC8 P273 and M274). While the methoxy group forms non-polar contacts with the pyrrolidine ring of P291, the phenyl 
ring of the inhibitor interacts (4.3 $\AA$ ) via either $\pi-\pi$ or cation- $\pi$ interaction with H292, depending upon the protonation state of the histidine (Figure 3B).

The smHDAC8 and hHDAC8 differ by one residue in their active sites, where hHDAC8 M274 is replaced by smHDAC8 H292. Since this latter residue is involved in inhibitor binding, we asked whether the smHDAC8-H292M mutant binds PCI-34051 in the same way as the wild-type (WT) enzyme. The crystal structure of PCI-34051 bound to the "humanized" smHDAC8-H292M mutant reveals that PCI34051 still adopts a L-shaped conformation when bound to the smHDAC8-H292M mutant, but this conformation is slightly different from the one adopted with the WT enzyme (Table S1; Figure S2A-C).

Specifically, PCI-34051 still lies over Y341 catalytic tyrosine but appears more centred in the pocket created by smHDAC8 Y341, F151 and the L6 loop. In contrast to the WT enzyme, the central indole group of PCI-34051 is axially rotated by an angle of $\sim 20^{\circ}$, which favors the positioning of the capping

methoxyphenyl group over the aromatic ring of Y341 (4.8 $\AA$ ), effecting nearly parallel $\left(8.9^{\circ}\right) \pi-\pi$ stacking. As a consequence, the PCI-34051 capping group interacts differently with the L6 loop than is observed with the WT enzyme, but still making close non-polar contacts with the aliphatic ring of P291 and the side chain of M292 in this loop (Figure S2A).

\section{Binding mode of NCC-149 to smHDAC8}

NCC-149 hydroxamate also binds in a canonical way to the catalytic zinc and active site residues, and the rest of the L-shaped inhibitor is turned towards and interacts with Y341 and the smHDAC8 L6 loop (Figure 3C). Specifically, the 1,2,3-triazole ring of the linker is oriented in a position (4.9 $\AA$ ) that is slightly off perpendicular $\left(\sim 83^{\circ}\right)$ to the aromatic ring of Y341, indicating their $\pi-\pi$ contacts. At the same time, the 1,2,3-triazole ring packs against L6 loop H292, which allows their mutual T-shaped $\left(\sim 67^{\circ}\right)$ aromatic interactions. In addition, and as observed for PCI-34051, the phenylthiomethyl capping group 
of NCC-149 is inserted in the small subpocket of the HDAC8 L6 loop, where it effects both upright $\left(\sim 76^{\circ}\right) \pi-\pi$ stacking with H292 and hydrophobic contacts (3.6 ̊̊) with P291.

We also solved the structure of NCC-149 bound to the smHDAC8-H292M mutant. Here, the hydroxamate and linker of NCC-149 bind very similarly to the smHDAC8-H292M mutant and to the WT enzyme, and show fewer conformational changes than observed with PCI-34051. Interestingly, the 1,2,3-triazole ring is closer to the L6 loop, where it interacts with M292 via a sulphur-aromatic interaction (3.7 A), suggesting a similar interaction with hHDAC8 (Figure S2D-F). This binding mode still favors T-shaped (82 $\left.{ }^{\circ}\right) \pi-\pi$ stacking between the 1,2,3-triazole and Y341 (4.7 $\AA$ ), as well as hydrophobic contacts between the internal benzene ring and the two phenylalanines, F151 and F216. However, the terminal phenylthiomethyl capping group of the inhibitor changes its position and is turned away from the L6 loop, lying in another binding subpocket formed by smHDAC8 K20 and F21 (L1 loop) and Y341 and F343 (L7 Loop) whose hydrophobic character is conserved in hHDAC8 (Figure S2D).

The conformational adaptation of NCC-149 to the smHDAC8-H292M selective pocket is eased by the intrinsically higher conformational flexibility of this inhibitor that allows the repositioning of its capping group. In the case of PCI-34051, which is more rigid as it contains only a one-atom hinge, a major part of the inhibitor had to be repositioned. Yet, these changes do not affect the major interaction of HDAC8selective inhibitors with the uncovered aromatic ring of the catalytic tyrosine and with residues of the L6 loop, showing the importance of these elements as key binding surfaces for these selective inhibitors.

\section{Selective inhibition of smHDAC8 over other human HDACs}

Previous work on the selective inhibition of smHDAC8 has yielded the development of an inhibitor series of 3-benzamido-benzohydroxamates that show strong selectivity for smHDAC8 and hHDAC8 over other human HDACs ${ }^{29}$. The structure of smHDAC8 with the simplest inhibitor of this series (1) revealed that 
the capping benzamido moiety lays over Y341 (3.9 Å), its benzene ring capping group further making non-polar contacts with the smHDAC8 L6 loop, notably with P291 (3.6 Å) (Figure 4A). This inhibitor also exploits smHDAC8-specific interactions with residues K20 and H292 (hHDAC8 K33 and M274) ${ }^{29}$.

Many of the 3-benzamido-benzohydroxamate inhibitors that were subsequently developed displayed higher potency than $\mathbf{1}$ in inhibiting smHDAC $8^{29}$. To understand the molecular basis of these observations, we have further solved the structures of smHDAC8 bound to several of these inhibitors (compounds 211) (Figure 4; Figure S3; Table S2; Table S3).

All these compounds showed a similar mode of binding to smHDAC8 as $\mathbf{1}$. Yet, slight differences in chemical composition impacted specific interactions, potentially relating to the differences in the $\mathrm{IC}_{50}$ values observed. Compounds 2-4 only have different substituents at the para position of the benzohydroxamate moiety compared to $\mathbf{1}$. These compounds bind very similarly to smHDAC8 as $\mathbf{1}$ (Figure S3). Their lower $\mathrm{IC}_{50}$ values most likely stem from the additional contacts of their substituent groups with F216, as well as the possible stabilization by these groups of the non-canonical geometry of the amide group of these inhibitors.

In the case of $\mathbf{5}$ and $\mathbf{6}$, which have respectively larger biphenyl and benzothiophene capping groups, these latter form more extensive hydrophobic contacts (3.5 $\AA$ ) with P291 (Figure 4B; Figure S3). In the case of $\mathbf{7}$, which only has an inverted internal amide compared to $\mathbf{1}$, the orientation and the length of the hydrogen bonds between 7 and smHDAC8 K20 and H292 appear more favorable for interaction (Figure S3).

Compounds 8-10 are particularly interesting since they also show a higher selectivity for smHDAC8 over hHDAC8 (3-, 4.5-, and 6-fold; respectively) (Figure 4C; Figure S3; Table S2). 8 has one additional methylene group between the internal amide and the phenyl capping group compared to $\mathbf{1}$. Compound $\mathbf{8}$ 
appears to bind less deeply in the pocket to maximize its interactions with Y341 and the L6 loop. This change is compatible with the presence of smHDAC8 H292, but would be sterically unfavourable with hHDAC8 M274, which possibly explains the weaker inhibition observed for the human enzyme.

Compounds 9 and 10 both have a dichlorophenyl rather than a phenyl capping group, and bind perfectly into the pocket formed by smHDAC8 Y341 and the L1 and L6 loops (Figure 4C; Figure S3). The presence of the halogen atoms in the capping group of $\mathbf{9 / 1 0}$ forces the inhibitor to be slightly tilted towards the L6 loop, where it forms cation- $\pi$ interaction (4.3 $\AA$ ) with smHDAC8 H292. The bulkier character of the dichlorophenyl capping group may complicate the adaptation of $\mathbf{9} / \mathbf{1 0}$ to the active site of hHDAC8.

Indeed, the predicted docking poses of $\mathbf{9}$ and $\mathbf{1 0}$ in hHDAC8 show that their dichlorophenyl capping groups are turned around $180^{\circ}$ in comparison to their position in smHDAC8 and effects van der Waals interactions with the hydrophobic residues of the L6 loop P273 and M274 (Figure S4). When comparing the obtained $\mathrm{IC}_{50}$ values of compounds $\mathbf{9 / 1 0}$ bearing a dichlorophenyl capping group with their parent counterparts $\mathbf{3 / 4}$, which have a phenyl capping group, it becomes clear that the observed selectivity of the former compounds for smHDAC8 over hHDAC8 arises from a significantly decreased inhibitory activity towards hHDAC8. Compared to compounds 3/4, compounds $\mathbf{9}$ and $\mathbf{1 0}$ show a 7- to 10-fold decrease in their inhibitory activities towards hHDAC8 (Table S2). To further explain the selectivity of the dichlorophenyl derivatives, we carried out 100 ns molecular dynamics (MD) simulations for smHDAC8/10 and hHDAC8/10 complexes, as well as for smHDAC8/4 and hHDAC8/4 complexes for comparison.

MD simulations of smHDAC8/4 crystal structure (Figure S5A) reveal a relatively stable binding mode, where the phenyl capping group is placed parallel to Y341 in the side pocket, forming $\pi$ - $\pi$ stacking interactions, in addition to cation- $\pi$ interactions with H292. During the MD simulation of smHDAC8/10 
crystal structure (Figure S5B), the dichlorophenyl capping group similarly remains in the side pocket. Despite the loss of the $\pi-\pi$ stacking interactions with Y341, compound $\mathbf{1 0}$ is still able to form strong cation- $\pi$ interactions with $\mathrm{H} 292$, which are stable throughout MD. This might explain why the dichlorophenyl derivative only shows a slight decrease in inhibitory activity as compared to compound 4.

Similarly, MD simulations of the hHDAC8/4 docking complex (Figure S5C) show that the phenyl capping group mostly remains stable in the side pocket, where it is placed perpendicular to Y306 of the side pocket, showing strong $\pi-\pi$ stacking interactions besides van der Waals interactions with M274. In contrast, our MD simulation of hHDAC8/10 structure indicates that the predicted binding mode of the dichlorophenyl derivative 10, where the capping phenyl ring is situated parallel to Y306, is not stable. Within less than $1 \mathrm{~ns}$ of the MD simulation time, the capping group flips by $90^{\circ}$ and is placed perpendicular to Y306 with the o-chloro substituent pointing towards the side chain of Y306. (Figure S5D). The $\pi-\pi$ stacking interactions with Y306, observed with compound 4 , are thus lost. Thus, a possible explanation for the decreased inhibitory activity of $\mathbf{1 0}$ for hHDAC8 as compared to compound $\mathbf{4}$ is that the dichlorophenyl capping group can only form weak van der Waals interactions between its o-chloro group and the side chain of Y306 and between its phenyl capping group and the side chain of M274.

We have used another benzohydroxamate inhibitor (11) which has an internal amine rather than an internal amide in its linker and shows a low nM IC 50 for hHDAC8 but only a low $\mu \mathrm{M} \mathrm{IC}_{50}$ for smHDAC8 (Table S2). The smHDAC8/11 structure reveals that this inhibitor does not form any strong interaction with K20 and H292 and its capping group interacts less extensively with the L6 loop (Figure S3; Figure S6). The inhibitor binds centrally into the pocket, where it forms hydrophobic contacts with F216 (3.4 $\AA$ ) and Y341 (3.7 $\AA$ ), in a conformation identical to that observed for PCI-34051 when bound to the smHDAC8-H292M mutant (Figure S6). This suggests that HDAC8-selective inhibitors bind more 
centrally in this pocket in hHDAC8 than in smHDAC8 due to the slight different physico-chemical properties of the active sites of these two proteins.

Finally, an unrelated compound, 12, which has a triazole linker, binds similarly to smHDAC8, interacting with Y341 and the L6 loop (Figure S3). This compound also adopts an L-shaped conformation to bind to the enzyme. 12 does not make direct contacts to K20 and H292, which might explain its higher IC 50 value for smHDAC8 (Table S2). This compound displays, however, a 4-fold higher potency for smHDAC8 over hHDAC8. Collectively, our results highlight how small chemical variations may be used to influence inhibition potency.

\section{Structural specificity of the HDAC8-selective pocket}

Our findings show an HDAC8 selective inhibition relying on the binding of the selective inhibitors to a pocket that forms a shallow groove and that we have termed HDAC8-selective pocket. This pocket is defined by the catalytic tyrosine side-chain (L7 loop), which forms the pocket bottom, and residues from the L6 loop and, to a lesser extent, of the L1 loop of HDAC8 that both form the sides of the pocket.

The HDAC8-selective pocket is highly specific to this enzyme. Indeed, in class IIa HDACs, the catalytic tyrosine is replaced by a histidine whose side chain is turned away from the active site and cannot provide the same interaction surface as HDAC8 catalytic tyrosine (Figure 5). The situation is different for HDAC isozymes 1, 2, 3, 6 and 10 (thereafter called HDAC1-3,6,10) that all have retained a catalytic tyrosine at the same position. Specifically, these isozymes have a L6 loop that displays a similar conformation to that observed in HDAC8 (Figure 5). L6 loops in HDAC1-3,6,10 however protrude slightly more over the catalytic tyrosine side chain than in the case of HDAC8 and could sterically perturb the binding of HDAC8-selective inhibitors (Figure 5). 
Yet, our results with the smHDAC8-H292M mutant show that HDAC8-selective inhibitors can adapt to small changes within the HDAC8-selective pocket and could potentially overcome a more protrusive L6 loop. However, another specific and conserved feature of HDAC1-3,6,10 is a larger L1 loop that extends towards the L6 loop. Notably, at the tip of the HDAC1-3,6,10 L1 loop, a proline (or an isoleucine residue in HDAC10) is present that forms hydrophobic interactions with L6 loop residues and the catalytic tyrosine, thus forming a lock over the catalytic tyrosine and preventing the formation of a pocket similar to the HDAC8-selective pocket (Figure 5).

In HDAC8, the L1 and L2 loops have been shown to display flexibility, being able to change their conformation to adapt to different inhibitors such as the large Largazole inhibitor and its analogues (Figure S7) ${ }^{40,41}$. This raises the question of whether the same L1 loop flexibility might exist in HDAC13,6,10. Current structural data on HDAC1-3,6,10, however, show that their L1 loops make much more extensive contacts with the rest of the enzyme, which most likely explains this lack of flexibility. Notably, the recent work on HDAC6/inhibitor complexes demonstrate that L1 loop conformational stability is important for the interactions of L1 loop residues with inhibitors capping groups to achieve HDAC6 selectivity inhibition ${ }^{42-46}$.

These major structural differences between HDAC8 and the other HDAC isozymes would explain how HDAC8 selective inhibition is achieved by inhibitors such as PCI-34051 and NCC-149. This is supported by docking studies of PCI-34051 and NCC-149 with other HDAC isozymes (Figure S8). These studies show that in case of HDAC1-3 and HDAC10, these inhibitors cannot reach properly and chelate the catalytic zinc ion. This is not as clear for the HDAC6 isozyme. Therefore, to assess the stability of the predicted docking poses and to further study the selectivity of PCI-34051 and NCC-149 towards HDAC8, we performed comparative 100 ns MD simulations on human HDAC6 and HDAC8 isoforms complexed with both inhibitors (Figure S8 and S9). In hHDAC8, PCI-34051 shows stable 
binding with an RMSD at $\sim 2 \AA$ compared to the predicted docking pose. The capping group shows little deviation and remains in the side pocket throughout the $100 \mathrm{~ns}$ MD simulation (Figure S9A). The same is observed for NCC-149, which displays a stable RMSD at 2 $\AA$ compared to the docking pose, except for a short increase in RMSD at 65-70 ns, with the capping group mostly remaining in the side pocket (Figure S10A).

In smHDAC8, PCI-34051 shows higher RMSD deviation at 3-4 $\AA$ compared to the crystal structure. The high RMSD arises from the movement of the capping group, which however remains in the side pocket throughout the simulation time (Figure S9B). The MD simulation of the smHDAC8/NCC-149 crystal structure yielded similar results as observed for the smHDAC8/PCI-34051 complex (Figure S10B).

In contrast, MD simulations of hHDAC6/PCI-34051 and hHDAC6/NCC-149 complexes, as predicted by docking, show high RMSD deviations for both ligands with RMSD values at $\sim 5$ and up to $7 \AA$, respectively (Figures S9C and S10C). Throughout the $100 \mathrm{~ns}$ simulation, the ligands maintain their chelation to the catalytic zinc ion. Meanwhile, the capping group of both ligands does not show any preferred conformation and remain surface exposed throughout the MD simulation, showing little interactions with the surrounding protein residues (Figure S9C and S10C). Collectively, the MD simulations confirm the hypothesis made upon docking and are in agreement with the biochemical, biophysical and crystallographic results obtained.

\section{Essential roles of L1 and L6 loops in HDAC8 catalysis and inhibition}

To further question the importance of HDAC8 L1 and L6 loops conformation in catalysis and inhibitor binding, we have performed a mutational analysis of these loops in HDAC8 and studied the effect of these mutations by biochemical, biophysical and structural means. Despite an identical number of 
residues, the HDAC8 L6 loop has a conformation that is slightly different from that adopted by the corresponding loops in HDAC1-3,6,10. This slight conformational change prevents L6 loop residues from protruding over the catalytic tyrosine (Figure 5).

Sequence and structural comparisons highlighted two residues that could, at first sight, be responsible for this specific conformation of HDAC8 L6 loop: hHDAC8 P273/smHDAC8 P291 and hHDAC8 C275/smHDAC8 R293. However, we could not exclude the possibility that larger rearrangements are required, and we therefore created several different mutants for both hHDAC8 and smHDAC8 (Figure 6A; Table S4).

First, specific point mutants (hHDAC8 P273R and P273R/C275G and smHDAC8 P291R and P291R/R293G) were made, where the residues were replaced by their HDAC1 counterparts. Second, we created mutants where we exchanged the HDAC8 L6 loop completely with that of HDAC1. We also constructed mutants where not only the L6 loop but also the L1 loop had been exchanged. Finally, triple mutants were also generated where a leucine (hHDAC8 L179/smHDAC8 L187) was replaced by an isoleucine, as observed in HDAC1, since this mutation could possibly facilitate an L6 loop conformational change (Figure 6A; Table S4).

Except for the point mutants, all smHDAC8 mutants turned out to be insoluble. In contrast, all hHDAC8 mutants were soluble. Surprisingly, activity assays showed that all mutants had drastically reduced activity (Table S4). While smHDAC8 single point mutants lost around one third of their activity, hHDAC8 point mutants or L6 loop replacement mutants showed around 10-fold activity loss. Mutants of hHDAC8 combining L6 loop replacement with either L1 loop replacement or the L179I point mutation showed a 50-fold loss of activity, and the triple mutant displayed almost no activity. 
We used Thermal Shift Assay experiments to assess whether the mutations affect the stability of the various mutants. All mutants only showed a decreased $\mathrm{Tm}$ of about $5^{\circ} \mathrm{C}$ compared to the WT enzymes but did not indicate partial or complete unfolding of the proteins (Table S4). Due to the residual activity of the mutants, we next measured the $\mathrm{IC}_{50}$ values for PCI-34051, NCC-149 and QSN for all mutants. All inhibitors showed significantly higher $\mathrm{IC}_{50}$ values indicative of a poorer inhibition capacity (Figure 6B; Table S5). This was most pronounced for inhibitor PCI-34051, with a more than 64-fold increase of the $\mathrm{IC}_{50}$ value for the triple HDAC8 mutant. The $\mathrm{IC}_{50}$ values for NCC-149 were also significantly increased, but not as much as for PCI-34051. QSN also displayed decreased inhibition, albeit to a much lesser extent, possibly mirroring only the slight stability decrease of the mutants but supporting our conclusions on the crucial role of L1 and L6 loops for HDAC8 selective inhibition.

To investigate the molecular basis of conformational changes of the L1 and L6 loops following mutations, we attempted to solve the structures of the different HDAC8 mutants in complex with PCI-34051, NCC149 and QSN. Although different mutants gave crystals in presence of some of these inhibitors, only the crystals obtained with the human HDAC8-mL6/QSN complex led to exploitable structural data (Table S6). To our knowledge this is the first structure of compound QSN bound to human HDAC8. Here again the capping group of the inhibitor is extensively involved in crystal packing and it is impossible to understand whether the binding conformation of QSN, which is different to that observed when bound to smHDAC8, represents a favored binding conformation in solution to hHDAC8 (Figure S11). Strikingly, the structure of this complex revealed that, despite the complete exchange of the L6 loop, the conformation of this loop remains as observed in hHDAC8 WT (Figure 6C). The hHDAC8 P273R replacement, however, leads to the partial occupation of the HDAC8-selective pocket by the side chain of the arginine replacing the proline (Figure 6). In fact, the only mutation common to all our smHDAC8 and hHDAC8 mutants is this proline to arginine change. In hHDAC1 and hHDAC3 this arginine is 
involved in inositol phosphate binding and is important for activity ${ }^{38,47,48}$. Our results therefore further highlight the importance of the L6 loop for class I HDACs activity and inhibitor binding and pinpoint differences between HDAC8 and the other members of this class that can be used for selective inhibition of the former enzyme.

\section{DISCUSSION AND CONCLUSIONS}

To date, HDAC8 is one of the most investigated HDAC for selective inhibition and numerous crystallographic studies have been performed on human HDAC8 in complex with, mostly, non-selective inhibitors to understand the molecular basis of the inhibition of this enzyme ${ }^{33,34,40,41,49-54}$. Many of these studies have revealed how HDAC8 active site residues and loops, notably the L1 and L2 loops, display flexibility, thus making the design of HDAC8 selective inhibitors a complicated task. In addition, in many structures, the bound inhibitor is involved in extensive crystallographic contacts, which makes difficult to assess whether the observed conformations of the inhibitor, notably of its capping group, but also potentially of the active site loops, are biologically favored.

Yet, HDAC8 selective inhibitors have been designed, showing that selective inhibition of this enzyme can be achieved. One of the most potent and best known HDAC8 selective inhibitor is PCI-34051 ${ }^{16}$. However, so far, the molecular basis for its selective inhibition remains poorly understood. We have previously shown that our smHDAC8 crystals can be used to look at inhibitor binding in a crystal latticeunbiased manner ${ }^{29,}$ 30, 55-58. Here, we have used this feature to look at HDAC8 selective inhibition using a large set of selective inhibitors of this enzyme, including PCI-34051.

Our results provide a comprehensive and detailed molecular view of HDAC8 selective inhibition, highlighting a specific HDAC8-selective pocket where selective inhibitors form preferential interactions with HDAC8 catalytic tyrosine and L6 loop residues. These results particularly emphasize the balance 
between chemical structure and inherent conformational flexibility of the inhibitors, with important implications for selective inhibition. In addition, the restricted conformation of HDAC8 L6 loop, compared to the more flexible character of the L1 loop, points out the importance of the L6 loop for selective inhibition. Moreover, the constrained conformation of the L7 loop that bears the catalytic tyrosine also appears as an essential feature of HDAC8 selective inhibition. Specifically, the position of the tyrosine side chain, which is locked by the interaction of its hydroxyl with the inhibitor's hydroxamate, most likely constrains the reported flexibility of the L7 loop ${ }^{59}$.

Interestingly, in class IIa HDACs the replacement of the catalytic tyrosine by a histidine creates a specific pocket at the position of the tyrosine side chain. This feature prevents HDAC8-selective inhibitors from binding to class IIa HDACs and has been exploited to design class IIa-selective inhibitors that cannot distinguish, however, between the different class IIa isozymes ${ }^{60}$.

In the case of HDAC1-3,6,10, although the catalytic tyrosine is conserved and the L6 loop is similar in these isozymes, the HDAC8-selective pocket is not present due to a larger L1 loop in HDAC1-3,6,10 that covers the catalytic tyrosine and forms a lock together with loop L6 over this selectivity pocket. The structures of HDAC6 in complex with HDAC6-selective inhibitors show that the capping group of these inhibitors interact with the proline at the tip of the L1 loop as well as with the preceding histidine residue. This mirrors the interaction observed between the HDAC8-selective inhibitors and the smHDAC8 L6 loop P291-H292 motif. Surprisingly, these inhibitors are selective for HDAC6 although the same motif is conserved in HDAC1-3 L1 loops that show similar conformations as HDAC6 L1 loop. Selective inhibition appears to rely on small structural differences between HDAC1-3 and HDAC6 and on small thermodynamic differences in selective inhibitor binding ${ }^{42-46}$. This parallels our study that demonstrate how selective inhibition can be affected by small changes in protein conformation and inhibitor composition. 
Interestingly, the HDAC8-selective pocket can adapt to bulkier inhibitors that can also interact with the back of the pocket, as observed for compound NCC-149 when bound to the smHDAC8-H292M mutant, thus potentially paving the way for the design of more potent HDAC8-selective inhibitors. Of note, the reduced conformational flexibility of compound PCI-34051, if it prevents this inhibitor from easily adapting to small changes within the HDAC8 active site, might also explain why this inhibitor is so selective for this enzyme.

The existence of a unique selective specific pocket in HDAC8 and the effects observed on activity when this pocket is partially occupied indicate that it might also be essential for the recognition of specific targets. Collectively, our results unravel the structural determinants underlying HDAC8 selective inhibition and pave the way toward the design of more potent HDAC selective inhibitors, the development of novel epigenetic drugs and the delineation of HDACs specific biological role through chemical biology approaches.

\section{EXPERIMENTAL SECTION}

\section{Small-molecule inhibitors.}

The inhibitors PCI-34051 and Quisinostat (QSN) were purchased from Selleck Chemicals (USA). The NCC-149 inhibitor was purchased from the Tokyo Chemical Company (Japan). The smHDAC8selective inhibitors were synthesized and characterized as described previously ${ }^{29,}{ }^{6}$ unless stated below. Compound 6 (3-(benzthiophene-7-carboxamido)-4-chlorobenzohydroxamate) was synthesized as follows (Figure S12A). Procedure. (a) Benzthiophene-7-carboxylic acid (1.4 mmol) was cooled to $0^{\circ} \mathrm{C}$ and then thionyl chloride ( $3 \mathrm{mmol}$ ) was added dropwise. The mixture was heated under reflux for 30 minutes. After evaporating the excess of thionyl chloride under vacuum, the obtained acid chloride was dissolved in dry THF $(50 \mathrm{ml})$ and added to a solution of methyl 3-amino-4-chlorobenzoate $(1.4 \mathrm{mmol})$ 
and DIPEA (3 mmol) in THF. The reaction was monitored by TLC. Subsequently, the solvent was evaporated under vacuum and the mixture was dissolved in ethyl acetate (50 ml) and washed with aq. sodium hydroxide solution. The organic layer was evaporated under reduced pressure and the product was purified by column chromatography (chloroform/ methanol, 99:1). (Yield: 88.8\%). (b) The obtained amide was dissolved in methanol (25 $\mathrm{ml})$ and $1 \mathrm{M}$ aq. sodium hydroxide solution $(10 \mathrm{ml})$ and heated to $50^{\circ} \mathrm{C}$ for $2 \mathrm{~h}$. The reaction was monitored by TLC. Subsequently, the solvent was evaporated under reduced pressure and the product was dissolved in ethyl acetate and washed with $1 \mathrm{M} \mathrm{HCl} \mathrm{solution,} \mathrm{the}$ organic layer was finally evaporated under reduced pressure. (Yield: 85.0\%). (c) The substituted benzoic acid derivative $(1 \mathrm{mmol})$ was dissolved in dry THF $(50 \mathrm{ml})$ and PyBOP $(1.2 \mathrm{mmol})$ was added. To the activated acid, a mixture of $\mathrm{NH}_{2} \mathrm{OTHP}(1.5 \mathrm{mmol})$ and DIPEA (2.5 mmol) in dry THF (5 ml) was added, and the reaction mixture was stirred overnight at room temperature. The solvent was evaporated under reduced pressure and the mixture was dissolved in ethyl acetate $(50 \mathrm{ml})$ and washed with aq. potassium hydrogen carbonate solution and brine. The organic layer was evaporated under reduced pressure and the product was purified by column chromatography (chloroform/methanol/TEA, 99.5:0.45:0.05). The obtained product was dissolved in THF and a catalytic amount of diluted $\mathrm{HCl}$ was added and was stirred at room temperature. The reaction was controlled by TLC. After that, the solvent was evaporated under reduced pressure and the mixture was dissolved in ethyl acetate $(50 \mathrm{ml})$ and washed with brine. The organic layer was evaporated under reduced pressure and the product was purified by column chromatography (chloroform/methanol/formic acid, 95:4.95:0.05). (Yield: 35\%). Analytical data. MS m/z: $345.23\left(\mathrm{Cl}^{35}\right) \mid 347.24\left(\mathrm{Cl}^{37}\right)[\mathrm{M}-\mathrm{H}]^{-} .{ }^{1} \mathrm{H}$ NMR (400 MHz, DMSO-d 6$) \delta 11.37(\mathrm{~s}, 1 \mathrm{H}), 10.42(\mathrm{~s}, 1 \mathrm{H})$, 9.14 (s, 1H), 8.22 (d, J = 7.3 Hz, 1H), 8.15 (d, J = $7.7 \mathrm{~Hz}, 1 \mathrm{H}), 8.02$ (s, 1H), 7.85 (d, J = 5.2 Hz, 1H), $7.74-7.63$ (m, 2H), 7.59 (t, J = $7.5 \mathrm{~Hz}, 1 \mathrm{H}), 7.53$ (d, J = 5.2 Hz, 1H). HRMS m/z: 369.0072 [M+Na] 
calculated: $\mathrm{C}_{16} \mathrm{H}_{11} \mathrm{~N}_{2} \mathrm{O}_{3} \mathrm{ClSNa}^{+}$369.0071. HPLC: rt 10.68 min (99.65\%). Yield: 120 mg; 0.35 mmol; $25 \%$.

Compound 12 (1-[5-chloro-2-(4-fluorophenoxy)phenyl]-N-hydroxy-1H-1,2,3-triazole-4-carboxamide) was synthetized as follows (Figure S12B). Procedure: (a) 2-Amino-4-chlorophenol (7.0 mmol) was dissolved in $1 \mathrm{M} \mathrm{HCl}(20 \mathrm{~mL})$. At $-5{ }^{\circ} \mathrm{C}$, a solution of sodium nitrite $(8.4 \mathrm{mmol})$ in water $(2 \mathrm{~mL})$ was added dropwise over a period of $5 \mathrm{~min}$. After stirring additional $5 \mathrm{~min}$, urea (50 mg) was added. Then the mixture was added to a cold solution of sodium azide $(14 \mathrm{mmol})$ and sodium acetate $(0.06 \mathrm{mmol})$ in water $(10 \mathrm{~mL})$. The resulting mixture was stirred for $2 \mathrm{~h}$ at $-5^{\circ} \mathrm{C}$. Then it was extracted with diethyl ether $(3 \times)$. The combined organic layers were dried $\left(\mathrm{Na}_{2} \mathrm{SO}_{4}\right)$, filtered, and the solvent was removed under reduced pressure. The residue was purified by flash column chromatography (cyclohexane/ethyl acetate = 10/1). (Yield: 86\%). (b) The obtained azide (3.0 mmol) was dissolved in a 1:1 mixture of water and tert-butyl alcohol (15 mL). Methyl propiolate (4.0 mmol), sodium ascorbate (0.20 mmol), and copper(II) sulfate pentahydrate $(0.04 \mathrm{mmol})$ were added and the mixture was stirred for $18 \mathrm{~h}$ at room temperature. Then water was added and the mixture was extracted with ethyl acetate $(3 \times)$. The combined organic layers were dried $\left(\mathrm{Na}_{2} \mathrm{SO}_{4}\right)$, filtered, and the solvent was removed under reduced pressure. The residue was purified by flash column chromatography (cyclohexane/ethyl acetate = 2/1). (Yield: 89\%). (c) A 100 $\mathrm{mL}$ round-bottom flask was charged with the obtained triazole (0.59 mmol), $\mathrm{Cu}(\mathrm{OAc})_{2}(0.59 \mathrm{mmol}), 4-$ fluorophenylboronic acid (0.71 mmol), and powdered $4 \AA$ molecular sieves. Then dichloromethane (4.5 $\mathrm{mL})$ was added. After the addition of triethylamine $(2.9 \mathrm{mmol})$, the reaction mixture was stirred at ambient temperature overnight. Then the suspension was filtered and the filtrate diluted with water and extracted with ethyl acetate $(3 \times)$. The combined organic layers were dried $\left(\mathrm{Na}_{2} \mathrm{SO}_{4}\right)$, filtered, and the solvent was removed under reduced pressure. The residue was purified by flash column chromatography (cyclohexane/ethyl acetate $=9 / 1 \rightarrow 3 / 1$ ). (Yield: 13\%). (d) A $5.4 \mathrm{M}$ solution of sodium methoxide in 
methanol $(1.1 \mathrm{mmol})$ was added to a solution of the obtained diphenyl ether $(0.11 \mathrm{mmol})$ and hydroxylamine hydrochloride $(0.55 \mathrm{mmol})$ in dry methanol $(3 \mathrm{~mL})$. The mixture was stirred at ambient temperature overnight. Then the solvent was removed under reduced pressure and the residue was purified by automatic flash column chromatography using a Biotage purification apparatus $(5 \% \rightarrow 50 \%$ ACN in $\mathrm{H}_{2} \mathrm{O}$, Biotage ${ }^{\circledR}$ SNAP KP-C18-HS 12 g). Fractions containing the desired product were combined, dried from acetonitrile under reduced pressure and then subjected to lyophilization. (Yield: 95\%).

Analytical data: ${ }^{1} \mathrm{H}$ NMR (DMSO-d 6$): \delta[\mathrm{ppm}]=7.02-7.06(\mathrm{~m}, 1 \mathrm{H}), 7.15-7.20$ (m, 2H), $7.22-7.28$ (m, 2H), $7.53-7.57$ (m, 1H), $7.87-7.90(\mathrm{~m}, 1 \mathrm{H}), 8.31$ (s, 1H); ${ }^{13} \mathrm{C}$ NMR $\left(\mathrm{DMSO}-\mathrm{d}_{6}\right): \delta[\mathrm{ppm}]=116.9$ (d, $J=23.7 \mathrm{~Hz}, 2 \mathrm{C}), 120.4$ (1C), 121.1 (d, $J=8.7 \mathrm{~Hz}, 2 \mathrm{C}), 123.1$ (1C), 125.7 (1C), 127.4 (1C), 128.8 (1C), 130.2 (1C), 147.1 (1C), 148.4 (1C), 151.3 (d, $J=2.4 \mathrm{~Hz}, 1 \mathrm{C}), 158.3$ (1C), 158.8 (d, $J=241 \mathrm{~Hz}$ 1C); HRMS (m/z): $[\mathrm{M}+\mathrm{H}]^{+}$calculated for $\mathrm{C}_{15} \mathrm{H}_{11} \mathrm{ClFN}_{4} \mathrm{O}_{3}$ : 349.0498, found: 349.0522; HPLC: $\mathrm{t}_{\mathrm{R}}=16.0$ min, purity $95.7 \%$.

\section{Purity of chemicals.}

The purity of all compounds used was assessed by HPLC. All synthesized compounds have a purity higher than 95\%. The purity for the PCI-34051 and Quisinostat (QSN) compounds purchased from Selleck Chemicals is $99.01 \%$ and $99.62 \%$, respectively, as stated by the manufacturer (see HPLC data in the following webpages: http:/www.selleckchem.com/products/pci-34051.html and http://www.selleckchem.com/products/JNJ-26481585.html). The purity for the NCC-149 compound purchased from the Tokyo Chemical Company is $96.00 \%$ as stated by the manufacturer (see http://www.tcichemicals.com/eshop/en/sg/commodity/H1340/). The HPLC traces for all other compounds are provided in Figure S13A-L). 


\section{Cloning, expression and purification of HDAC8 proteins.}

The full-length cDNA constructs (WT and mutants) for hHDAC8 and smHDAC8 were amplified by polymerase-chain reaction (PCR) and cloned into bacterial expression vectors. The hHDAC8 (WT and mutant) gene was inserted between the NdeI and BamHI restriction sites of the pnEA-3HT expression vector $^{61}$, where it is in frame with a sequence coding for a $\mathrm{N}$-terminal poly-histidine affinity purification tag followed by thioredoxin and a protease 3C cleavage site. The smHDAC8 (WT and mutant) gene was cloned between the NdeI and BamHI restriction sites of the pnEA-tH expression vector ${ }^{61}$ and is in frame with a sequence coding for a C-terminal thrombin cleavage site followed by a poly-histidine affinity purification tag.

For wild-type smHDAC8 overproduction, a modified protocol was used compared to that published previously. Expression was carried out in BL21(DE3) cells in 2xLB medium. Cultures were grown and induced at $37^{\circ} \mathrm{C}$ with $0.7 \mathrm{mM}$ IPTG in the presence of $100 \mu \mathrm{M} \mathrm{ZnCl}$. After overnight incubation at $37^{\circ} \mathrm{C}$, cells were harvested and resuspended in lysis buffer A (150 mM NaCl, $50 \mathrm{mM}$ Tris pH 8.0). Lysis was done by sonication, the lysate was clarified by centrifugation. The supernatant was loaded onto Talon Superflow Metal Affinity Resin (Clontech) pre-equilibrated with the lysis buffer A. The his-tagged protein was released from the Talon resin by thrombin protease treatment in buffer B (50 mM KCl, 10 mM Tris pH 8.0) and subsequently loaded onto a 16/60 Superdex 200 gel filtration column (GE Healthcare) pre-equilibrated with buffer C (50 mM KCl, 10 mM Tris-HCl pH 8.0 and 2 mM DTT). Peak fractions were concentrated with an Amicon Ultra centrifugal filter unit. This protocol yielded three times more protein for the wild type enzyme. smHDAC8 mutants could not be purified using this protocol and their overproduction was carried out as described initially ${ }^{30,62}$.

hHDAC8 overproduction was carried out in Escherichia coli BL21(DE3) cells in 2xLB medium. Culture induction was done at $23^{\circ} \mathrm{C}$ by adding $0.5 \mathrm{mM}$ final isopropyl-1-thio- $\beta$-D-galactopyranoside (IPTG, 
Euromedex), in the presence of $100 \mu \mathrm{M} \mathrm{ZnCl}_{2}$. Harvested bacteria were re-suspended in lysis buffer (50 $\mathrm{mM} \mathrm{KCl,} 10 \mathrm{mM}$ Tris-HCl pH 8.0) and lysed by sonication. The lysate was clarified by centrifugation (17,500 rpm, $50 \mathrm{~min}, 4^{\circ} \mathrm{C}$, Sorvall Lynx 6000 Thermo Scientific). The supernatant was loaded onto Talon Superflow Metal Affinity Resin (Clontech) pre-equilibrated with lysis buffer. The his-thioredoxin-tagged protein was released from the Talon resin by 3C protease treatment and subsequently loaded onto a 16/60 Superdex 200 gel filtration column (GE Healthcare) pre-equilibrated with the purification buffer (50 mM KCl, $10 \mathrm{mM}$ Tris-HCl pH 8.0, and 0.5 mM TCEP). The recombinant protein was concentrated with an Amicon Ultra centrifugal filter unit.

\section{Mutagenesis experiments.}

The mutant constructs were generated using standard PCR-based nested protocols and inserted into the corresponding expression vectors. The L1 and L6 loops exchange mutants were designed based on structural comparison. The hHDAC1 L6 sequence S265LSGDRLGC was introduced instead of the T268 $_{2}$ AGDPMCS sequence in hHDAC8, to create the hHDAC8-mL6 mutant. In the second step, the hHDAC1 L1 sequence $\mathrm{Y}_{23}$ YGQGHPMK was introduced instead of the L31AKI sequence in hHDAC8mL6, to create the double hHDAC8-mL1/mL6 mutant. Finally, the triple mutant hHDAC8$\mathrm{mL1} / \mathrm{mL} 6 / \mathrm{L} 179 \mathrm{I}$ was generated by the introduction of an isoleucine residue in the L179 position, using the double hHDAC8-mL1/mL6 mutant as a template. smHDAC8 mutants were produced as described for hHDAC8 mutants. Sequences replaced in smHDAC8 were the same as the ones replaced in hHDAC8.

\section{Crystallization and $\mathrm{X}$-ray data collection.}

Diffraction-quality crystals of the native smHDAC8 enzyme were obtained at $17^{\circ} \mathrm{C}$ after 3 days by mixing equal volumes of smHDAC8 $(2.5 \mathrm{mg} / \mathrm{ml})$ with reservoir solution composed of 21\% PEG 3350 (Fluka) and $0.05 \mathrm{M} \mathrm{Na}^{+} / \mathrm{K}^{+}$L-tartrate, and crystallized using the hanging-drop vapor diffusion technique. 
After 3 days, grown crystals were soaked for 20 hours in mother liquor supplemented with the corresponding inhibitor (10 mM final concentration of the inhibitor, preparation from a $100 \mathrm{mM}$ stock in N,N-dimethylformamide (DMF) or DMSO). Crystals used for X-ray data collection were briefly transferred in reservoir solution supplemented with 22\% glycerol and flash-frozen in liquid nitrogen.

Co-crystallization of hHDAC8-mL6 together with QSN (Quisinostat) inhibitor was performed using the hanging-drop vapor diffusion technique. The hHDAC8-mL6/QSN complex was formed by incubating the hHDAC8-mL6 mutant protein (5 mg/mL) with QSN (5 mM resuspended in DMF) at $4^{\circ} \mathrm{C}$ for $1 \mathrm{~h}$. Diffraction-quality crystals were obtained at $20^{\circ} \mathrm{C}$ after 3-4 days by mixing equal volumes of the hHDAC8-mL6/QSN complex with reservoir solution composed of 20\% polyethylene glycol 3350 (Fluka), 0.2 $\mathrm{M} \mathrm{KNO}_{3}$ and $0.1 \mathrm{M}$ Bis-tris propane $\mathrm{pH}$ 7.5. Crystals used for X-ray data collection were briefly transferred in reservoir solution supplemented with 22\% glycerol and flash-frozen in liquid nitrogen. Crystallographic data obtained in this project were collected at $100 \mathrm{~K}$ on SOLEIL beamline PROXIMA1, ESRF beamlines ID30b, ID29 and ID23 and SLS PX beamlines.

\section{Structure determination, model building and refinement.}

The crystallographic data were processed and scaled using HKL200063 or XDS ${ }^{64}$. Phases for smHDAC8/inhibitor complexes were obtained by molecular replacement followed by rigid body refinement against smHDAC8 native structure as a model (4BZ5). The initial models were refined through several cycles of manual building using $\operatorname{Coot}^{65}$ and automated refinement with Phenix ${ }^{66}$. The structure of hHDAC8-mL6 complexed with QSN was solved by molecular replacement with Phenix ${ }^{66}$ using the hHDAC8 structure (PDB 1T67) as a search model. The final models were validated using tools provided in Coot $^{65}$ and Molprobity ${ }^{67}$. Visualization of structural data was done with Pymol (The PyMOL Molecular Graphics System, Version 2.0 Schrödinger, LLC), and two-dimensional diagrams summarizing molecular interaction between inhibitors and HDAC8 enzymes were prepared with the help 
of the LigPlot program ${ }^{68}$. Atomic coordinates and structure factors of the smHDAC8 and hHDAC8-mL6 complexes were deposited in the Protein Data Bank under the PDB codes 6HQY, 6HRQ, 6HSH, 6HSF, 6HSG, 6HSZ, 6HT8, 6HTG, 6HTH, 6HTI, 6HTT, 6HTZ, 6HU0, 6HU1, 6HU2, 6HU3, 6HSK.

\section{HDAC activity and inhibition assays.}

Catalytic activity and inhibition assays of smHDAC8 and hHDAC8 were performed as described earlier $^{30}$. Briefly, the hHDAC8 and smHDAC8 activity testing was carried out with the HDAC8 Fluorimetric Drug Discovery Kit (Fluor de Lys(R)- HDAC8, BML-KI178) from Enzo Life Sciences, according to the manufacturer's instructions with a substrate concentration of $50 \mu \mathrm{M}$. Fluorescence was measured in a plate reader (BMG Polarstar) with excitation at $\lambda=390 \mathrm{~nm}$ and emission at $\lambda=460 \mathrm{~nm}$. IC50 values were determined with OriginPro (version 9.0.0, Northampton, Massachusetts).

\section{Studies on Schistosoma mansoni in culture.}

The effects of PCI-34051 and NCC-149 compounds on the viability of S. mansoni schistosomula (Puerto-Rican strain) were tested using a microscopy-based assay, as described previously ${ }^{69}$. In brief, schistosomula (2,000 per well), prepared by standard mechanical transformation from cercaria ${ }^{70}$, were maintained in 6-well plates in M199 medium kept at pH 7.4 with $10 \mathrm{mM}$ HEPES and supplemented at $37^{\circ} \mathrm{C}$ in a humid atmosphere containing $5 \% \mathrm{CO}$. Three different concentrations of inhibitors $(25,50$ and $100 \mu \mathrm{M}$ ) were tested, the inhibitors were dissolved in DMSO, and the culture medium was refreshed each day. The assessment of parasite mortality was carried out after microscopic examination, based on three criteria: a granular appearance, tegumental defects and the absence of motility. At least 300 schistosomula were observed at each time point for each condition and results were expressed as a percentage of viable larvae remaining. Three biological replicates (different batches of larvae) were examined in duplicate for each condition. 
The stability of adult worm pairs and egg production were assayed as previously described ${ }^{70}$. Adult worm pairs were obtained from infected golden hamsters (Mesocricetus auratus) by perfusion, washed in M199 medium and ten pairs placed in $2 \mathrm{~mL}$ of M199 buffered complete medium (as for schistosomula above) in each well of a 6-well culture plate. Worms were maintained in culture for 5 days at $37^{\circ} \mathrm{C}$ (humid atmosphere, $5 \% \mathrm{CO}_{2}$ ) before the addition of HDAC8-selective inhibitors, the application of DMSO alone served as a negative control experiment. Both the culture medium and the inhibitors were refreshed daily. The number of couples remaining as pairs was determined daily by microscopy and the medium of each well containing eggs laid by the couples was recovered and centrifuged to allow eggs to be counted under the microscope. Three biological replicate experiments were performed in triplicate.

\section{TUNEL assay.}

Detection of DNA strand breaks in inhibitor-treated S. mansoni schistosomula was done using the terminal deoxynucleotidyl transferase dUTP nick-end labelling (TUNEL) method using the In Situ Cell Death Detection Kit TMR Red (Roche). The method designed for cell suspensions was followed as described in the manufacturer's instructions with few modifications. Briefly, 2,000 schistosomula were treated with $100 \mu \mathrm{M}$ PCI-34051 or NCC-149 for $96 \mathrm{~h}$, in 6-well plates containing $2 \mathrm{~mL}$ of complete medium. The treatment with DMSO alone served as negative control experiment. After $96 \mathrm{~h}$ incubation, culture media were removed and the schistosomula were centrifuged (1,000 rpm, $2 \mathrm{~min}$ ), washed three times in PBS buffer, and then fixed in 2\% formaldehyde for 60 min. Schistosomula were afterwards washed once more in PBS and permeabilization solution (0.1\% Triton X-100, 0.1\% sodium citrate) was added for 10 min on ice. Labeling of schistosomula with 4',6-diamidino-2-phenylindole (DAPI) and TMR Red dUTP was performed according to the manufacturer's instructions and TUNEL-positive parasites were observed by fluorescence using an AxioImager Z1-Apotome microscope (Zeiss).

\section{Isothermal titration calorimetry (ITC).}


ITC experiments were done at $25^{\circ} \mathrm{C}$ using a PEAQ microcalorimeter (Malvern Instruments). All protein samples were purified in the same ITC buffer $(50 \mathrm{mM} \mathrm{KCl,} 10 \mathrm{mM}$ Tris $\mathrm{pH}=8.0$ and $0.5 \mathrm{mM}$ TCEP). In a typical experiment, aliquots of $2.0 \mu \mathrm{l}$ of HDAC8 protein sample at $200 \mu \mathrm{M}$ were injected into an inhibitor solution at $20 \mu \mathrm{M}$ placed in the ITC cell. Blank experiments were used to retrieve signal due to solvent (DMSO or DMF) dilution into ITC buffer. Data were analysed with PEAQ-ITC Analysis Software (Malvern Instruments) and with Affinimeter (Software 4 Science Developments).

\section{Differential scanning fluorimetry.}

Thermal stability of HDAC8 proteins was analysed by a label-free differential scanning fluorimetry (DSF) approach using a Prometheus NT.48 instument (NanoTemper Technologies). Briefly, the shift of intrinsic tryptophan fluorescence of HDAC8 proteins upon gradual temperature-triggered unfolding (temperature gradient 20 to $95^{\circ} \mathrm{C}$ ) was monitored by detecting the emission fluorescence at 330 and 350 $\mathrm{nm}$. The measurements were carried out nanoDSF-grade high sensitivity glass capillaries (NanoTemper Technologies) at a heating rate of $1^{\circ} \mathrm{C} / \mathrm{min}$. Protein melting points $(\mathrm{Tm})$ were inferred from the first derivative of the ratio of tryptophan emission intensities at 330 and $350 \mathrm{~nm}$. Finally, the $\Delta \mathrm{Tm}$ value of an HDAC8 protein for a particular inhibitor was calculated as the difference between the Tm values of the inhibitor-bound and inhibitor-free proteins. All the assays were done in triplicate.

\section{Docking experiments.}

Crystal structures of hHDAC1-3, hHDAC8 and hHDAC6-CD2 for docking as well as zHDAC10 for homology modelling of hHDAC10 were downloaded from Protein Data Bank (corresponding PDB IDs 4BKX, 4LXZ, 4A69, 2V5X, 5EDU, 5TD7). Also the zHDAC6 CD1-2 structure with Nexturastat A(PDB ID 5G0J) was downloaded from PDB in order to retrieve conserved water molecules for docking to hHDAC6-CD2. Specifically, water molecule W2141 coordinating the zinc ion was extracted from 
zHDAC6 and inserted in the superimposed structure of hHDAC6-CD2. This water molecule was used to stabilize the monodentate coordination of the zinc ion by the inhibitor, as is observed for hydroxamic acids with bulky aromatic linkers in HDAC6 X-ray structures. The homology model of human HDAC10 was built on zebrafish HDAC10 structure ${ }^{71}$ using MODELLER version $9.11^{72}$. Structures of inhibitors were generated in MOE version 2014.09 (Molecular Operating Environment (MOE), 2014.09; Chemical Computing Group Inc., 1010 Sherbooke St. West, Suite 910, Montreal, QC, Canada, H3A 2R7, 2014).

Protein and inhibitor structures were further prepared for docking in Schrödinger Suite (Schrödinger Suite 2014-2: Maestro version 9.8, Protein Preparation Wizard; Epik version 2.8, Glide version 9.8, Schrödinger, LLC, New York, NY, USA, 2014). Human HDAC1-3,6,10 were prepared using the Protein Preparation Wizard tool. Hydrogen atoms and missing amino acid residues side chains were added. Solvent molecules were removed except for two conserved water molecules: one near the catalytic zinc ion and another above the zinc coordinating histidine residue (H180, hHDAC8 numbering). Next, hydrogen bond networks and amino acid residues protonation states and tautomers were optimized. Finally, protein models were subjected to energy minimization using OPLS-2005 force field with default settings. Inhibitor structures were prepared with LigPrep and ConfGen tools. Namely, the tautomeric forms and stereoisomers were created and energy minimization was performed with OPLS force field using Ligprep. Conformers were generated with default settings (fast) and energy minimization of the output conformations was performed using ConfGen.

Molecular docking was performed using Glide from the Schrödinger Suite. Receptor grids were generated using default settings. The Standard Precision docking protocol with default settings without any constraints was used, except the number of docking poses for post-docking minimization per ligand was increased to 20 and the maximal number of output poses per ligand was increased to 2.

\section{Molecular dynamics simulations.}


Initial coordinates of the smHDAC8/inhibitor complexes were taken from the newly resolved crystal structures of smHDAC8 in complex with PCI-34051, NCC-149, and compounds 4 and 10. Coordinates of PCI-34051, NCC-149, and compounds 4 and 10 in complex with hHDAC8, as well as PCI-34051 and NCC-149 in complex with HDAC6 were taken from the obtained docking poses described above. All ligands were generated in the negatively-charged hydroxamate form, and the protein residues were charged at $\mathrm{pH} 7$, where the His residue coordinating the negatively charged O-atom of the hydroxamate was assigned a positive charge (HIP residue). Water molecules and $\mathrm{K}^{+}$ions were kept.

MD simulations were performed using Amber16 package (AMBER 2016, UCSF, San Francisco, California, USA, 2016). Force field parameters for all ligands were assigned using the Antechamber package and AM1-BCC atomic charges ${ }^{73}$, 74 . The TLEaP module of Amber16, the ff03 force field ${ }^{75}$ and the General Amber Force Field (GAFF) ${ }^{76}$ were applied to the protein residues and ligand, respectively, and the 12-6-4 LJ-type nonbonded model parameters ${ }^{77}$ for divalent ions in SPC/E water model was used for the catalytic $\mathrm{Zn}^{2+}$ ion. The complex structures were solvated in an octahedral periodic box of SPC/E water molecules ${ }^{78}$ at a margin of $10 \AA$ and the system was neutralized using $\mathrm{Na}^{+}$counter ions.

The system was first subjected to consecutive steps of minimization. The first step encompassed 3000 iterations (first 1000 steepest descent and then 2000 conjugate gradient), where only solvent atoms were minimized, while restraining the protein and ligand atoms as well as the $\mathrm{Zn}^{2+}$ ion to their initial coordinates with a force constant of $10 \mathrm{kcal} . \mathrm{mol}^{-1} . \AA^{-2}$. In the second step, the whole system was minimized with no restraints using 4000 iterations (first 2000 steepest descent and then 2000 conjugate gradient). The system was then heated to the production temperature of $300 \mathrm{~K}$ through $100 \mathrm{ps}$ of MD, while keeping the complex atoms (protein, ligand, and zinc ion) restrained with a force constant of 10 kcal.mol ${ }^{-1} . \AA^{-2}$ to prevent large structural deviations. Constant volume periodic boundary was set to equilibrate the temperature of the system by Langevin thermostat using a collision frequency of $2 \mathrm{ps}^{-1}$. 
The system was subsequently subjected to a pressure equilibration routine for $100 \mathrm{ps}$ at $300 \mathrm{~K}$, where a constant pressure of 1 bar was applied.

Finally, a production run of 100 ns was simulated at constant temperature (300 K) using the Langevin thermostat with a collision frequency of $2 \mathrm{ps}^{-1}$. Constant pressure periodic boundary was used to maintain the pressure of the system at 1 bar using isotropic position scaling with a relaxation time of 2 ps. During the temperature equilibration and MD routines a non-bonded cut-off distance of $10.0 \AA$ was used by applying the Particle Mesh Ewald (PME) method ${ }^{79}$ for long-range electrostatic interactions. The SHAKE algorithm $^{80}$ was applied to constrain all bonds involving hydrogen. A time step of 2 fs was used for equilibration and the production of MD. All simulations were run using PMEMD.cuda implementation from AMBER16 on CUDA-enabled NVIDIA graphics processing units (GPUs).

The trajectories were analyzed using the CPPTRAJ module of AMBER and visualized using VMD. Plots were generated using the R package, and the pictures using PYMOL.

\section{PAINS Filter.}

All the herein described compounds were filtered for pan-assay interference compounds (PAINS). For this purpose, PAINS1, PAINS2, and PAINS3 filters, as implemented in Schrödinger's Canvas module (Schrödinger Suite 2014-2: Canvas version 2.0), were employed. None of the compounds was flagged as PAINS.

\section{ANCILLARY INFORMATION}

\section{Supporting Information.}

The supporting information contains additional figures and tables. An Excel file also provides the Molecular Formula Strings for all compounds. This material is available free of charge via the Internet at http://pubs.acs.org. 
The supporting information includes:

Figure S1. Fitness analysis of schistosomes treated with inhibitors PCI-34051 and NCC-149.

Figure S2. Structures of PCI-34051 and NCC-149 bound to the smHDAC8-H292M mutant.

Figure S3. Close-up view of HDAC8-selective inhibitors bound to smHDAC8.

Figure S4. Docking results of compounds $\mathbf{9}$ and $\mathbf{1 0}$ bound to human HDAC8.

Figure S5. Snapshots and RMSD plots of MD simulations of smHDAC8/4, smHDAC8/10, hHDAC8/4, and hHDAC8/10 complexes.

Figure S6. Comparison of the binding modes of compound $\mathbf{1 1}$ and PCI-34051 to smHDAC8.

Figure S7. Structural plasticity and flexibility of human HDAC8 L1 and L2 loops.

Figure S8. Docking poses of PCI-34051 and NCC-149 in different HDAC isozymes.

Figure S9. Snapshots and RMSD plots of MD simulations of HDAC/PCI-34051 complexes.

Figure S10. Snapshots and RMSD plots of MD simulations of HDAC/NCC-149 complexes.

Figure S11. Binding mode of Quisinostat (QSN) in the structure of the human HDAC8-mL6/QSN complex.

Figure S12. Synthesis pathways.

Figure S13. HPLC traces compounds 1-12.

Table S1. Data collection and refinement statistics for the structures of smHDAC8 WT and H292M mutant bound to PCI-34051, NCC-149 and Quisinostat.

Table S2. IC 50 values for HDAC8-selective inhibitors on different HDACs. 
Table S3. Data collection and refinement statistics for the structures of smHDAC8 bound to compounds 1-12.

Table S4. HDAC8 mutants and activity measurements

Table S5. IC50 values for hHDAC8 mutants with PCI-34051, NCC-149 and Quisinostat (QSN).

Table S6. Data collection and refinement statistics for the structure of human HDAC8-mL6/Quisinostat (QSN) complex.

\section{PDB ID codes.}

Atomic coordinates and structure factors of the smHDAC8 and hHDAC8-mL6 complexes were deposited in the Protein Data Bank under the PDB ID codes 6HQY, 6HRQ, 6HSH, 6HSF, 6HSG, 6HSZ, 6HT8, 6HTG, 6HTH, 6HTI, 6HTT, 6HTZ, 6HU0, 6HU1, 6HU2, 6HU3, 6HSK. Authors will release the atomic coordinates and experimental data upon article publication.

\section{Corresponding Author Information}

* Correspondence should be addressed to:

Martin Marek (martin.marek@recetox.muni.cz)

Christophe Romier (omier@igbmc.fr)

\section{Present/Current Author Addresses}

†Current address: Loschmidt Laboratories, Department of Experimental Biology \& RECETOX, Faculty of Science, Masaryk University, Kamenice 5/A13, 62500 Brno, Czech Republic.

\section{Author Contributions}

¥These authors contributed equally to this work. 
M.M., T.B.S., E.R.M. and C.R. solved the HDAC8-inhibitor crystal structures, constructed and biophysically characterized the HDAC8 mutants. J.L. and R.J.P. performed anti-parasitic studies. C.D.V., M.M. and E.E. performed and interpreted the ITC experiments. T.S. synthesized the NCC-149 inhibitor. D.K. and R.H synthesized inhibitor 12. T.H., J.M. and D.R. carried out docking studies, synthesized and characterized smHDAC8 inhibitors. A.C., K.S. and M.J. performed enzymatic activity and inhibition assays. M.M., R.J.P, M.J., W.S. and C.R. designed experiments, analyzed data and wrote the paper.

All authors have given approval to the final version of the manuscript.

\section{Acknowledgment}

This work and the authors of this manuscript have been supported by funding from the European Union's Seventh Framework Programme for research, technological development and demonstration under grant agreements nos. 241865 (SEtTReND) and 602080 (A-ParaDDisE) as well as the German Research Foundation (DFG) to WS (Si868/13-1) and MJ (Ju295/13-1). This study was supported by the grant ANR-10-LABX-0030-INRT, a French State fund managed by the Agence Nationale de la Recherche under the frame program Investissements d'Avenir ANR-10-IDEX-0002-02. CR, MM, TBS, ERM and RJP are supported by institutional funds from the Centre National de la Recherche Scientifique (CNRS), the Institut National de la Santé et de la Recherche Médicale (INSERM), the Université de Strasbourg, the Institut Pasteur de Lille and the Université de Lille. The authors acknowledge the support and the use of resources of the French Infrastructure for Integrated Structural Biology FRISBI ANR-10-INBS-05 and of Instruct-ERIC. We wish to thank members of the ESRF-EMBL joint structural biology groups, the SOLEIL, and the Swiss Light Source (SLS) synchrotrons for the use of their beamline facilities and for help during data collection. We are grateful to Alastair McEwen (IGBMC) for his kind assistance during X-ray data collections. 


\section{Abbreviations used}

HDAC, Histone Deacetylase; HDAC1-3,6,10, HDAC1, HDAC2, HDAC3, HDAC6 and HDAC10.

\section{REFERENCES}

1. Choudhary, C.; Weinert, B. T.; Nishida, Y.; Verdin, E.; Mann, M. The growing landscape of lysine acetylation links metabolism and cell signalling. Nat Rev Mol Cell Biol 2014, 15, 536-550.

2. Drazic, A.; Myklebust, L. M.; Ree, R.; Arnesen, T. The world of protein acetylation. Biochim Biophys Acta 2016, 1864, 1372-1401.

3. Verdin, E.; Ott, M. 50 years of protein acetylation: from gene regulation to epigenetics, metabolism and beyond. Nat Rev Mol Cell Biol 2015, 16, 258-264.

4. Haberland, M.; Montgomery, R. L.; Olson, E. N. The many roles of histone deacetylases in development and physiology: implications for disease and therapy. Nat Rev Genet 2009, 10, 32-42.

5. Marmorstein, R.; Zhou, M. M. Writers and readers of histone acetylation: structure, mechanism, and inhibition. Cold Spring Harb Perspect Biol 2014, 6, a018762.

6. Seto, E.; Yoshida, M. Erasers of histone acetylation: the histone deacetylase enzymes. Cold Spring Harb Perspect Biol 2014, 6, a018713.

7. Musselman, C. A.; Lalonde, M. E.; Cote, J.; Kutateladze, T. G. Perceiving the epigenetic landscape through histone readers. Nat Struct Mol Biol 2012, 19, 1218-1227.

8. Arrowsmith, C. H.; Bountra, C.; Fish, P. V.; Lee, K.; Schapira, M. Epigenetic protein families: a new frontier for drug discovery. Nat Rev Drug Discov 2012, 11, 384-400.

9. Campbell, R. M.; Tummino, P. J. Cancer epigenetics drug discovery and development: the challenge of hitting the mark. J Clin Invest 2014, 124, 64-69. 
10. Dawson, M. A.; Kouzarides, T. Cancer epigenetics: from mechanism to therapy. Cell 2012, 150, 1227.

11. Falkenberg, K. J.; Johnstone, R. W. Histone deacetylases and their inhibitors in cancer, neurological diseases and immune disorders. Nat Rev Drug Discov 2014, 13, 673-691.

12. Jones, P. A.; Issa, J. P.; Baylin, S. Targeting the cancer epigenome for therapy. Nat Rev Genet 2016, 17, 630-641.

13. West, A. C.; Johnstone, R. W. New and emerging HDAC inhibitors for cancer treatment. J Clin Invest 2014, 124, 30-39.

14. Chan, T. S.; Tse, E.; Kwong, Y. L. Chidamide in the treatment of peripheral T-cell lymphoma. Onco Targets Ther 2017, 10, 347-352.

15. Maolanon, A. R.; Madsen, A. S.; Olsen, C. A. Innovative strategies for selective inhibition of histone deacetylases. Cell Chem Biol 2016, 23, 759-768.

16. Balasubramanian, S.; Ramos, J.; Luo, W.; Sirisawad, M.; Verner, E.; Buggy, J. J. A novel histone deacetylase 8 (HDAC8)-specific inhibitor PCI-34051 induces apoptosis in T-cell lymphomas. Leukemia 2008, 22, 1026-1034.

17. Suzuki, T.; Muto, N.; Bando, M.; Itoh, Y.; Masaki, A.; Ri, M.; Ota, Y.; Nakagawa, H.; Iida, S.; Shirahige, K.; Miyata, N. Design, synthesis, and biological activity of NCC149 derivatives as histone deacetylase 8-selective inhibitors. ChemMedChem 2014, 9, 657-664.

18. Suzuki, T.; Ota, Y.; Ri, M.; Bando, M.; Gotoh, A.; Itoh, Y.; Tsumoto, H.; Tatum, P. R.; Mizukami, T.; Nakagawa, H.; Iida, S.; Ueda, R.; Shirahige, K.; Miyata, N. Rapid discovery of highly potent and 
selective inhibitors of histone deacetylase 8 using click chemistry to generate candidate libraries. $J$ Med Chem 2012, 55, 9562-9575.

19. Chakrabarti, A.; Melesina, J.; Kolbinger, F. R.; Oehme, I.; Senger, J.; Witt, O.; Sippl, W.; Jung, M. Targeting histone deacetylase 8 as a therapeutic approach to cancer and neurodegenerative diseases. Future Med Chem 2016, 8, 1609-1634.

20. Chakrabarti, A.; Oehme, I.; Witt, O.; Oliveira, G.; Sippl, W.; Romier, C.; Pierce, R. J.; Jung, M. HDAC8: a multifaceted target for therapeutic interventions. Trends Pharmacol Sci 2015, 36, 481-492.

21. Ramos, T. L.; Sanchez-Abarca, L. I.; Redondo, A.; Hernandez-Hernandez, A.; Almeida, A. M.; Puig, N.; Rodriguez, C.; Ortega, R.; Preciado, S.; Rico, A.; Muntion, S.; Porras, J. R. G.; Del Canizo, C.; Sanchez-Guijo, F. HDAC8 overexpression in mesenchymal stromal cells from JAK2+ myeloproliferative neoplasms: a new therapeutic target? Oncotarget 2017, 8, 28187-28202.

22. Deardorff, M. A.; Bando, M.; Nakato, R.; Watrin, E.; Itoh, T.; Minamino, M.; Saitoh, K.; Komata, M.; Katou, Y.; Clark, D.; Cole, K. E.; De Baere, E.; Decroos, C.; Di Donato, N.; Ernst, S.; Francey, L. J.; Gyftodimou, Y.; Hirashima, K.; Hullings, M.; Ishikawa, Y.; Jaulin, C.; Kaur, M.; Kiyono, T.; Lombardi, P. M.; Magnaghi-Jaulin, L.; Mortier, G. R.; Nozaki, N.; Petersen, M. B.; Seimiya, H.; Siu, V. M.; Suzuki, Y.; Takagaki, K.; Wilde, J. J.; Willems, P. J.; Prigent, C.; Gillessen-Kaesbach, G.; Christianson, D. W.; Kaiser, F. J.; Jackson, L. G.; Hirota, T.; Krantz, I. D.; Shirahige, K. HDAC8 mutations in Cornelia de Lange syndrome affect the cohesin acetylation cycle. Nature 2012, 489, 313317.

23. Deardorff, M. A.; Porter, N. J.; Christianson, D. W. Structural aspects of HDAC8 mechanism and dysfunction in Cornelia de Lange syndrome spectrum disorders. Protein Sci 2016, 25, 1965-1976. 
24. Kaiser, F. J.; Ansari, M.; Braunholz, D.; Concepcion Gil-Rodriguez, M.; Decroos, C.; Wilde, J. J.; Fincher, C. T.; Kaur, M.; Bando, M.; Amor, D. J.; Atwal, P. S.; Bahlo, M.; Bowman, C. M.; Bradley, J. J.; Brunner, H. G.; Clark, D.; Del Campo, M.; Di Donato, N.; Diakumis, P.; Dubbs, H.; Dyment, D. A.; Eckhold, J.; Ernst, S.; Ferreira, J. C.; Francey, L. J.; Gehlken, U.; Guillen-Navarro, E.; Gyftodimou, Y.; Hall, B. D.; Hennekam, R.; Hudgins, L.; Hullings, M.; Hunter, J. M.; Yntema, H.; Innes, A. M.; Kline, A. D.; Krumina, Z.; Lee, H.; Leppig, K.; Lynch, S. A.; Mallozzi, M. B.; Mannini, L.; McKee, S.; Mehta, S. G.; Micule, I.; Care4Rare Canada, C.; Mohammed, S.; Moran, E.; Mortier, G. R.; Moser, J. A.; Noon, S. E.; Nozaki, N.; Nunes, L.; Pappas, J. G.; Penney, L. S.; Perez-Aytes, A.; Petersen, M. B.; Puisac, B.; Revencu, N.; Roeder, E.; Saitta, S.; Scheuerle, A. E.; Schindeler, K. L.; Siu, V. M.; Stark, Z.; Strom, S. P.; Thiese, H.; Vater, I.; Willems, P.; Williamson, K.; Wilson, L. C.; University of Washington Center for Mendelian, G.; Hakonarson, H.; Quintero-Rivera, F.; Wierzba, J.; Musio, A.; Gillessen-Kaesbach, G.; Ramos, F. J.; Jackson, L. G.; Shirahige, K.; Pie, J.; Christianson, D. W.; Krantz, I. D.; Fitzpatrick, D. R.; Deardorff, M. A. Loss-of-function HDAC8 mutations cause a phenotypic spectrum of Cornelia de Lange syndrome-like features, ocular hypertelorism, large fontanelle and X-linked inheritance. Hum Mol Genet 2014, 23, 2888-2900.

25. Dasgupta, T.; Antony, J.; Braithwaite, A. W.; Horsfield, J. A. HDAC8 inhibition blocks SMC3 deacetylation and delays cell cycle progression without affecting cohesin-dependent transcription in MCF7 cancer cells. J Biol Chem 2016, 291, 12761-12770.

26. Hsieh, C. L.; Ma, H. P.; Su, C. M.; Chang, Y. J.; Hung, W. Y.; Ho, Y. S.; Huang, W. J.; Lin, R. K. Alterations in histone deacetylase 8 lead to cell migration and poor prognosis in breast cancer. Life Sci 2016, 151, 7-14. 
27. Lopez, G.; Bill, K. L.; Bid, H. K.; Braggio, D.; Constantino, D.; Prudner, B.; Zewdu, A.; Batte, K.; Lev, D.; Pollock, R. E. HDAC8, A potential therapeutic target for the treatment of malignant peripheral nerve sheath tumors (MPNST). PLoS One 2015, 10, e0133302.

28. Zhang, K.; Lu, Y.; Jiang, C.; Liu, W.; Shu, J.; Chen, X.; Shi, Y.; Wang, E.; Wang, L.; Hu, Q.; Dai, Y.; Xiong, B. HDAC8 functions in spindle assembly during mouse oocyte meiosis. Oncotarget 2017, 8, 20092-20102.

29. Heimburg, T.; Chakrabarti, A.; Lancelot, J.; Marek, M.; Melesina, J.; Hauser, A. T.; Shaik, T. B.; Duclaud, S.; Robaa, D.; Erdmann, F.; Schmidt, M.; Romier, C.; Pierce, R. J.; Jung, M.; Sippl, W. Structure-based design and synthesis of novel inhibitors targeting HDAC8 from Schistosoma mansoni for the treatment of schistosomiasis. J Med Chem 2016, 59, 2423-2435.

30. Marek, M.; Kannan, S.; Hauser, A. T.; Moraes Mourao, M.; Caby, S.; Cura, V.; Stolfa, D. A.; Schmidtkunz, K.; Lancelot, J.; Andrade, L.; Renaud, J. P.; Oliveira, G.; Sippl, W.; Jung, M.; Cavarelli, J.; Pierce, R. J.; Romier, C. Structural basis for the inhibition of histone deacetylase 8 (HDAC8), a key epigenetic player in the blood fluke Schistosoma mansoni. PLoS Pathog 2013, 9, e1003645.

31. Estiu, G.; West, N.; Mazitschek, R.; Greenberg, E.; Bradner, J. E.; Wiest, O. On the inhibition of histone deacetylase 8. Bioorg Med Chem 2010, 18, 4103-4110.

32. Krennhrubec, K.; Marshall, B. L.; Hedglin, M.; Verdin, E.; Ulrich, S. M. Design and evaluation of 'linkerless' hydroxamic acids as selective HDAC8 inhibitors. Bioorg Med Chem Lett 2007, 17, 28742878.

33. Somoza, J. R.; Skene, R. J.; Katz, B. A.; Mol, C.; Ho, J. D.; Jennings, A. J.; Luong, C.; Arvai, A.; Buggy, J. J.; Chi, E.; Tang, J.; Sang, B. C.; Verner, E.; Wynands, R.; Leahy, E. M.; Dougan, D. R.; Snell, G.; Navre, M.; Knuth, M. W.; Swanson, R. V.; McRee, D. E.; Tari, L. W. Structural snapshots 
of human HDAC8 provide insights into the class I histone deacetylases. Structure 2004, 12, 1325 1334.

34. Tabackman, A. A.; Frankson, R.; Marsan, E. S.; Perry, K.; Cole, K. E. Structure of 'linkerless' hydroxamic acid inhibitor-HDAC8 complex confirms the formation of an isoform-specific subpocket. J Struct Biol 2016, 195, 373-378.

35. Arts, J.; King, P.; Marien, A.; Floren, W.; Belien, A.; Janssen, L.; Pilatte, I.; Roux, B.; Decrane, L.; Gilissen, R.; Hickson, I.; Vreys, V.; Cox, E.; Bol, K.; Talloen, W.; Goris, I.; Andries, L.; Du Jardin, M.; Janicot, M.; Page, M.; van Emelen, K.; Angibaud, P. JNJ-26481585, a novel "second-generation" oral histone deacetylase inhibitor, shows broad-spectrum preclinical antitumoral activity. Clin Cancer Res 2009, 15, 6841-6851.

36. Venugopal, B.; Baird, R.; Kristeleit, R. S.; Plummer, R.; Cowan, R.; Stewart, A.; Fourneau, N.; Hellemans, P.; Elsayed, Y.; McClue, S.; Smit, J. W.; Forslund, A.; Phelps, C.; Camm, J.; Evans, T. R.; de Bono, J. S.; Banerji, U. A phase I study of quisinostat (JNJ-26481585), an oral hydroxamate histone deacetylase inhibitor with evidence of target modulation and antitumor activity, in patients with advanced solid tumors. Clin Cancer Res 2013, 19, 4262-4272.

37. Dubois, F.; Caby, S.; Oger, F.; Cosseau, C.; Capron, M.; Grunau, C.; Dissous, C.; Pierce, R. J. Histone deacetylase inhibitors induce apoptosis, histone hyperacetylation and up-regulation of gene transcription in Schistosoma mansoni. Mol Biochem Parasitol 2009, 168, 7-15.

38. Watson, P. J.; Millard, C. J.; Riley, A. M.; Robertson, N. S.; Wright, L. C.; Godage, H. Y.; Cowley, S. M.; Jamieson, A. G.; Potter, B. V.; Schwabe, J. W. Insights into the activation mechanism of class I HDAC complexes by inositol phosphates. Nat Commun 2016, 7, 11262. 
39. Lauffer, B. E.; Mintzer, R.; Fong, R.; Mukund, S.; Tam, C.; Zilberleyb, I.; Flicke, B.; Ritscher, A.; Fedorowicz, G.; Vallero, R.; Ortwine, D. F.; Gunzner, J.; Modrusan, Z.; Neumann, L.; Koth, C. M.; Lupardus, P. J.; Kaminker, J. S.; Heise, C. E.; Steiner, P. Histone deacetylase (HDAC) inhibitor kinetic rate constants correlate with cellular histone acetylation but not transcription and cell viability. J Biol Chem 2013, 288, 26926-26943.

40. Cole, K. E.; Dowling, D. P.; Boone, M. A.; Phillips, A. J.; Christianson, D. W. Structural basis of the antiproliferative activity of largazole, a depsipeptide inhibitor of the histone deacetylases. J Am Chem Soc 2011, 133, 12474-12477.

41. Decroos, C.; Clausen, D. J.; Haines, B. E.; Wiest, O.; Williams, R. M.; Christianson, D. W. Variable active site loop conformations accommodate the binding of macrocyclic largazole analogues to HDAC8. Biochemistry 2015, 54, 2126-2135.

42. Hai, Y.; Christianson, D. W. Histone deacetylase 6 structure and molecular basis of catalysis and inhibition. Nat Chem Biol 2016, 12, 741-747.

43. Porter, N. J.; Mahendran, A.; Breslow, R.; Christianson, D. W. Unusual zinc-binding mode of HDAC6-selective hydroxamate inhibitors. Proc Natl Acad Sci U S A 2017, 114, 13459-13464.

44. Miyake, Y.; Keusch, J. J.; Wang, L.; Saito, M.; Hess, D.; Wang, X.; Melancon, B. J.; Helquist, P.; Gut, H.; Matthias, P. Structural insights into HDAC6 tubulin deacetylation and its selective inhibition. Nat Chem Biol 2016, 12, 748-754.

45. Porter, N. J.; Osko, J. D.; Diedrich, D.; Kurz, T.; Hooker, J. M.; Hansen, F. K.; Christianson, D. W. Histone deacetylase 6-selective inhibitors and the influence of capping groups on hydroxamate-zinc denticity. J Med Chem 2018, 61, 8054-8060. 
46. Porter, N. J.; Wagner, F. F.; Christianson, D. W. Entropy as a driver of selectivity for inhibitor binding to histone deacetylase 6. Biochemistry 2018, 57, 3916-3924.

47. Millard, C. J.; Watson, P. J.; Celardo, I.; Gordiyenko, Y.; Cowley, S. M.; Robinson, C. V.; Fairall, L.; Schwabe, J. W. Class I HDACs share a common mechanism of regulation by inositol phosphates. Mol Cell 2013, 51, 57-67.

48. Watson, P. J.; Fairall, L.; Santos, G. M.; Schwabe, J. W. R. Structure of HDAC3 bound to corepressor and inositol tetraphosphate. Nature 2012, 481, 335-340.

49. Dowling, D. P.; Gantt, S. L.; Gattis, S. G.; Fierke, C. A.; Christianson, D. W. Structural studies of human histone deacetylase 8 and its site-specific variants complexed with substrate and inhibitors. Biochemistry 2008, 47, 13554-13563.

50. Dowling, D. P.; Gattis, S. G.; Fierke, C. A.; Christianson, D. W. Structures of metal-substituted human histone deacetylase 8 provide mechanistic inferences on biological function. Biochemistry 2010, 49, 5048-5056.

51. Gantt, S. M.; Decroos, C.; Lee, M. S.; Gullett, L. E.; Bowman, C. M.; Christianson, D. W.; Fierke, C. A. General base-general acid catalysis in human histone deacetylase 8. Biochemistry 2016, 55, 820832.

52. Vannini, A.; Volpari, C.; Filocamo, G.; Casavola, E. C.; Brunetti, M.; Renzoni, D.; Chakravarty, P.; Paolini, C.; De Francesco, R.; Gallinari, P.; Steinkuhler, C.; Di Marco, S. Crystal structure of a eukaryotic zinc-dependent histone deacetylase, human HDAC8, complexed with a hydroxamic acid inhibitor. Proc Natl Acad Sci U S A 2004, 101, 15064-15069. 
53. Vannini, A.; Volpari, C.; Gallinari, P.; Jones, P.; Mattu, M.; Carfi, A.; De Francesco, R.; Steinkuhler, C.; Di Marco, S. Substrate binding to histone deacetylases as shown by the crystal structure of the HDAC8-substrate complex. EMBO Rep 2007, 8, 879-884.

54. Whitehead, L.; Dobler, M. R.; Radetich, B.; Zhu, Y.; Atadja, P. W.; Claiborne, T.; Grob, J. E.; McRiner, A.; Pancost, M. R.; Patnaik, A.; Shao, W.; Shultz, M.; Tichkule, R.; Tommasi, R. A.; Vash, B.; Wang, P.; Stams, T. Human HDAC isoform selectivity achieved via exploitation of the acetate release channel with structurally unique small molecule inhibitors. Bioorg Med Chem 2011, 19, 46264934.

55. Bayer, T.; Chakrabarti, A.; Lancelot, J.; Shaik, T. B.; Hausmann, K.; Melesina, J.; Schmidtkunz, K.; Marek, M.; Erdmann, F.; Schmidt, M.; Robaa, D.; Romier, C.; Pierce, R. J.; Jung, M.; Sippl, W. Synthesis, crystallization studies, and in vitro characterization of cinnamic acid derivatives as smhdac8 inhibitors for the treatment of schistosomiasis. ChemMedChem 2018, 13, 1517-1529.

56. Heimburg, T.; Kolbinger, F. R.; Zeyen, P.; Ghazy, E.; Herp, D.; Schmidtkunz, K.; Melesina, J.; Shaik, T. B.; Erdmann, F.; Schmidt, M.; Romier, C.; Robaa, D.; Witt, O.; Oehme, I.; Jung, M.; Sippl, W. Structure-based design and biological characterization of selective histone deacetylase 8 (HDAC8) inhibitors with anti-neuroblastoma activity. J Med Chem 2017, 60, 10188-10204.

57. Simoben, C. V.; Robaa, D.; Chakrabarti, A.; Schmidtkunz, K.; Marek, M.; Lancelot, J.; Kannan, S.; Melesina, J.; Shaik, T. B.; Pierce, R. J.; Romier, C.; Jung, M.; Sippl, W. A novel class of Schistosoma mansoni histone deacetylase 8 (HDAC8) inhibitors identified by structure-based virtual screening and in vitro testing. Molecules 2018, 23, e566.

58. Stolfa, D. A.; Marek, M.; Lancelot, J.; Hauser, A. T.; Walter, A.; Leproult, E.; Melesina, J.; Rumpf, T.; Wurtz, J. M.; Cavarelli, J.; Sippl, W.; Pierce, R. J.; Romier, C.; Jung, M. Molecular basis for the 
antiparasitic activity of a mercaptoacetamide derivative that inhibits histone deacetylase 8 (HDAC8) from the human pathogen Schistosoma mansoni. J Mol Biol 2014, 426, 3442-3453.

59. Porter, N. J.; Christianson, N. H.; Decroos, C.; Christianson, D. W. Structural and Functional Influence of the glycine-rich loop G(302)GGGY on the catalytic tyrosine of histone deacetylase 8 . Biochemistry 2016, 55, 6718-6729.

60. Burli, R. W.; Luckhurst, C. A.; Aziz, O.; Matthews, K. L.; Yates, D.; Lyons, K. A.; Beconi, M.; McAllister, G.; Breccia, P.; Stott, A. J.; Penrose, S. D.; Wall, M.; Lamers, M.; Leonard, P.; Muller, I.; Richardson, C. M.; Jarvis, R.; Stones, L.; Hughes, S.; Wishart, G.; Haughan, A. F.; O'Connell, C.; Mead, T.; McNeil, H.; Vann, J.; Mangette, J.; Maillard, M.; Beaumont, V.; Munoz-Sanjuan, I.; Dominguez, C. Design, synthesis, and biological evaluation of potent and selective class IIa histone deacetylase (HDAC) inhibitors as a potential therapy for Huntington's disease. J Med Chem 2013, 56, 9934-9954.

61. Diebold, M. L.; Fribourg, S.; Koch, M.; Metzger, T.; Romier, C. Deciphering correct strategies for multiprotein complex assembly by co-expression: application to complexes as large as the histone octamer. J Struct Biol 2011, 175, 178-188.

62. Marek, M.; Shaik, T. B.; Duclaud, S.; Pierce, R. J.; Romier, C. Large-scale overproduction and purification of recombinant histone deacetylase 8 (HDAC8) from the human-pathogenic flatworm Schistosoma mansoni. Methods Mol Biol 2016, 1436, 109-118.

63. Otwinowski, Z.; Minor, W. Processing of x-ray diffraction data collected in oscillation mode. Methods Enzymol 1997, 276, 307-326.

64. Kabsch, W. Xds. Acta Crystallogr D Biol Crystallogr 2010, 66, 125-132. 
65. Emsley, P.; Cowtan, K. Coot: model-building tools for molecular graphics. Acta Crystallogr D Biol Crystallogr 2004, 60, 2126-2132.

66. Adams, P. D.; Afonine, P. V.; Bunkoczi, G.; Chen, V. B.; Davis, I. W.; Echols, N.; Headd, J. J.; Hung, L. W.; Kapral, G. J.; Grosse-Kunstleve, R. W.; McCoy, A. J.; Moriarty, N. W.; Oeffner, R.; Read, R. J.; Richardson, D. C.; Richardson, J. S.; Terwilliger, T. C.; Zwart, P. H. PHENIX: a comprehensive Python-based system for macromolecular structure solution. Acta Crystallogr D Biol Crystallogr 2010, 66, 213-221.

67. Chen, V. B.; Arendall, W. B., 3rd; Headd, J. J.; Keedy, D. A.; Immormino, R. M.; Kapral, G. J.; Murray, L. W.; Richardson, J. S.; Richardson, D. C. MolProbity: all-atom structure validation for macromolecular crystallography. Acta Crystallogr D Biol Crystallogr 2010, 66, 12-21.

68. Wallace, A. C.; Laskowski, R. A.; Thornton, J. M. LIGPLOT: a program to generate schematic diagrams of protein-ligand interactions. Protein Eng 1995, 8, 127-134.

69. Vanderstraete, M.; Gouignard, N.; Cailliau, K.; Morel, M.; Lancelot, J.; Bodart, J. F.; Dissous, C. Dual targeting of insulin and venus kinase Receptors of Schistosoma mansoni for novel antischistosome therapy. PLoS Negl Trop Dis 2013, 7, e2226.

70. Ramalho-Pinto, F. J.; Gazzinelli, G.; Howells, R. E.; Mota-Santos, T. A.; Figueiredo, E. A.; Pellegrino, J. Schistosoma mansoni: defined system for stepwise transformation of cercaria to schistosomule in vitro. Exp Parasitol 1974, 36, 360-372.

71. Hai, Y.; Shinsky, S. A.; Porter, N. J.; Christianson, D. W. Histone deacetylase 10 structure and molecular function as a polyamine deacetylase. Nat Commun 2017, 8, 15368.

72. Sali, A.; Blundell, T. L. Comparative protein modelling by satisfaction of spatial restraints. $J$ Mol Biol 1993, 234, 779-815. 
73. Jakalian, A.; Bush, B. L.; Jack, D. B.; Bayly, C. I. Fast, efficient generation of high-quality atomic charges. AM1-BCC model: I. method. Journal of Computational Chemistry 2000, 21, 132-146.

74. Jakalian, A.; Jack, D. B.; Bayly, C. I. Fast, efficient generation of high-quality atomic charges. AM1BCC model: II. parameterization and validation. J Comput Chem 2002, 23, 1623-1641.

75. Duan, Y.; Wu, C.; Chowdhury, S.; Lee, M. C.; Xiong, G.; Zhang, W.; Yang, R.; Cieplak, P.; Luo, R.; Lee, T.; Caldwell, J.; Wang, J.; Kollman, P. A point-charge force field for molecular mechanics simulations of proteins based on condensed-phase quantum mechanical calculations. J Comput Chem 2003, 24, 1999-2012.

76. Wang, J.; Wolf, R. M.; Caldwell, J. W.; Kollman, P. A.; Case, D. A. Development and testing of a general amber force field. J Comput Chem 2004, 25, 1157-1174.

77. Li, P.; Merz, K. M., Jr. Taking into account the ion-induced dipole interaction in the nonbonded model of ions. J Chem Theory Comput 2014, 10, 289-297.

78. Berendsen, H. J. C.; Grigera, J. R.; Straatsma, T. P. The missing term in effective pair potentials. Journal of Physical Chemistry 1987, 91, 6269-6271.

79. Darden, T.; York, D.; Pedersen, L. Particle Mesh Ewald - an N.Log(N) method for Ewald sums in large systems. Journal of Chemical Physics 1993, 98, 10089-10092.

80. Ryckaert, J. P.; Ciccotti, G.; Berendsen, H. J. C. Numerical-integration of cartesian equations of motion of a system with constraints - molecular-dynamics of N-alkanes. Journal of Computational Physics 1977, 23, 327-341. 


\section{FIGURES LEGENDS}

Figure 1. HDAC8 inhibition and binding by inhibitors PCI-34051, NCC-149 and QSN. (A) Chemical structures of PCI-34051, NCC-149 and Quisinostat (QSN). (B) IC $\mathrm{I}_{50}$ values and binding affinities/thermodynamic parameters of PCI-34051, NCC-149 and QSN for human HDAC8 (hHDAC8) and Schistosoma mansoni HDAC8 (smHDAC8).

Figure 2. ITC measured affinities and thermodynamic signatures of PCI-34051, NCC-149 and Quisinostat (QSN) binding to hHDAC8 and smHDAC8. ITC profiles of the titration of hHDAC8 (A-C) and smHDAC8 (D-F) with PCI-34051 (A,D), NCC-149 (B,E) and QSN (C,F). Top panels, titration data of the enzymes into the corresponding inhibitor solution. DP, Differential power. Bottom panels, integrated heat measurements for the titration enzyme with the corresponding inhibitor.

Figure 3. Structural characterization of QSN, PCI-34051 and NCC-149 binding to smHDAC8 (PDB IDs 6HSH, 6HQY, 6HRQ) (A-C). Binding modes of QSN (A), PCI-34051 (B) and NCC-149 (C) to smHDAC8. Upper panels: Simulated annealing omit electron density map contoured at $2 \sigma$ for the inhibitors when bound to smHDAC8. Middle panels: Binding mode of QSN, PCI-34051 and NCC-149 in the active site of smHDAC8. The inhibitors and important residues are shown as sticks. smHDAC8 L6 loop is colored green. The catalytic zinc ion is shown as orange sphere. Zinc coordination and hydrogen bonds are shown as dashed lines. Only HDAC8-selective inhibitors PCI-34051 and NCC-149 adopt an L-shaped conformation and interact with the catalytic tyrosine and the L6 loop. Lower panels: Two dimensional illustrations of binding. Zinc coordination, hydrogen bonds and aromatic interactions are shown as dashed lines. The corresponding distances between the atoms and/or chemical groups are given in $\AA$. Hydrophobic contacts are shown by grey arcs with spokes radiating toward the atoms involved. 
Figure 4. Structural characterization of 1, 6 and 9 HDAC8-selective inhibitors binding to smHDAC8 (PDB IDs 5FUE, 6HTI, 6HU0). (A-C) Binding modes of compounds 1 (A), 6 (B) and 9 (C) to smHDAC8. Upper panels: Simulated annealing omit electron density map contoured at $2 \sigma$ for $\mathbf{1 ,} 6$ and $\mathbf{9}$ when bound to smHDAC8. Middle panels: Binding mode of $\mathbf{1 , 6}$ and $\mathbf{9}$ in the active site of smHDAC8. Lower panels: Two dimensional illustrations of binding. Representations, coloring and display of interactions are as in Figure 3.

Figure 5. Structural delineation of HDAC8 selective inhibition. (A-D) Close-up views shown as ribbon and sticks of the superposed structures of (A) smHDAC8, smHDAC8-H292M and hHDAC8 (PDB IDs 6HQY, 6HSF, 1T67), (B) hHDAC4 and hHDAC7 (PDB IDs 5A2S, 3C0Z), (C) hHDAC1, hHDAC2 and hHDAC3 (PDB IDs 4BKX, 4LXZ, 4A69), and (D) zebrafish zHDAC6-(catalytic domain1)CD1 and zHDAC6-CD2, and zHDAC10 (PDB IDs 5G0J, 5TD7). The catalytic zinc is shown as orange sphere. HDAC8-selective inhibitor PCI-34051 is shown in (A) as light blue sticks when bound in HDAC8selective pocket. In the other HDACs this pocket is not formed since residues from L1 and L6 loops are protruding and forming a lock over the catalytic tyrosine (other class I and class IIb HDACs) or its replacement histidine (class IIa HDACs). (E) Surface representation of the pocket accommodating the linker and capping groups of the HDAC8-selective inhibitors. The PCI-34051 inhibitor is represented as sticks and lays on the catalytic tyrosine (purple). The pocket walls are formed by residues from the L1 (yellow) and L6 (green) loops. (F, G, H) Surface representation of the same region in hHDAC4 (PDB ID 5A2S) (F), hHDAC3 (PDB ID 4A69) (G), and hHDAC6-CD2 (PDB ID 5EDU) (H) using the same color code as in (E). In these latter HDACs, L1 and L6 loop residues interact and form a L1-L6 lock over the pocket.

Figure 6. HDAC8 mutants inhibition by PCI-34051, NCC-149 and QSN, and structure of the human HDAC8-mL6/QSN complex. (A) Structure-based sequence alignment of L1, L4 and L6 loop sequences 
from various HDACs. Red boxes show the regions that have been swapped between HDAC1 and HDAC8 in the mutational analysis. (B) IC 50 values for PCI-34051, NCC-149 and QSN on human HDAC8 WT and mutants. Assays were done in triplicate. Error bars represent the SD. (C) Close-up view of the hHDAC8-selective pocket in the human HDAC8-mL6/QSN complex (left panel; PDB ID 6HSK) compared to WT hHDAC8 bound to PCI-34051 (right panel; model based on PDB IDs 1 T67 and 6HSF) and the superposition of the two structures (middle panel). The side chain of the arginine R273 (from the hHDAC8-mL6/QSN complex) replacing P273 (from WT hHDAC8) binds into the HDAC8-selective pocket where PCI-34051 (and the other HDAC8-selective inhibitors) were shown to bind. This mutation, which is the only common mutation to all mutants used, is most likely responsible for the general decrease of activity observed for these mutants. 


\section{Figure 1.}

A
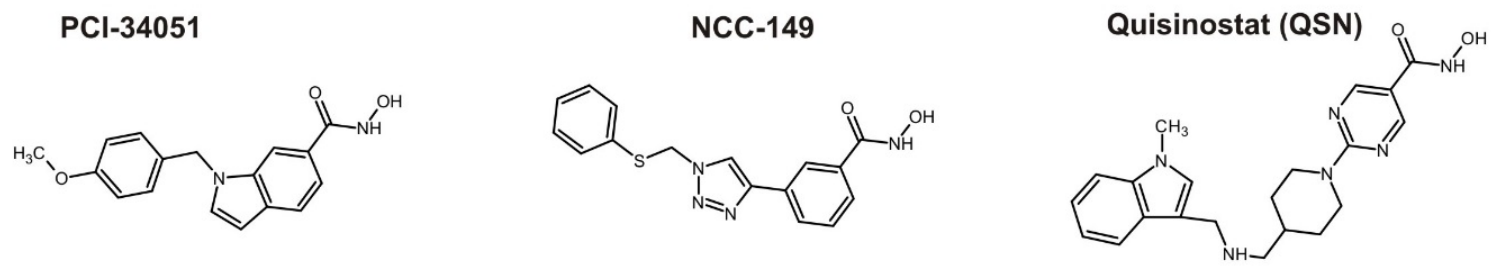

B

\begin{tabular}{|l|l|l|l|l|l|l|}
\hline Enzyme & Inhibitor & $\mathrm{IC}_{50}(\mathrm{nM})^{\star}$ & $\mathrm{Kd}(\mathrm{nM})$ & $\Delta \mathrm{G}(\mathrm{kcal} / \mathrm{mol})$ & $\Delta \mathrm{H}(\mathrm{kcal} / \mathrm{mol})$ & $-\mathrm{T} \Delta \mathrm{S}(\mathrm{kcal} / \mathrm{mol})$ \\
\hline hHDAC8 & PCI-34051 & $77.7 \pm 18.1$ & $75.1 \pm 6.9$ & -9.7 & -11.0 & 1.3 \\
\hline hHDAC8 & NCC-149 & $44 \pm 4.9$ & $89.6 \pm 18.4$ & -9.6 & -10.3 & 0.7 \\
\hline hHDAC8 & QSN & $64.4 \pm 3.4$ & $22.7 \pm 3.7$ & -10.4 & -12.7 & 2.3 \\
\hline smHDAC8 & PCI-34051 & $435.8 \pm 61$ & $367 \pm 40.7$ & -8.8 & -9.1 & -0.3 \\
\hline smHDAC8 & NCC-149 & $95.4 \pm 9.3$ & $81.7 \pm 7.0$ & -9.7 & -9.5 & -0.15 \\
\hline smHDAC8 & QSN & $303.4 \pm 39$ & $28.4 \pm 3.3$ & -10.3 & -8.8 & -1.5 \\
\hline
\end{tabular}

* IC50 measurements were done in triplicate. 
Figure 2.

A

hHDAC8+PCl-34051
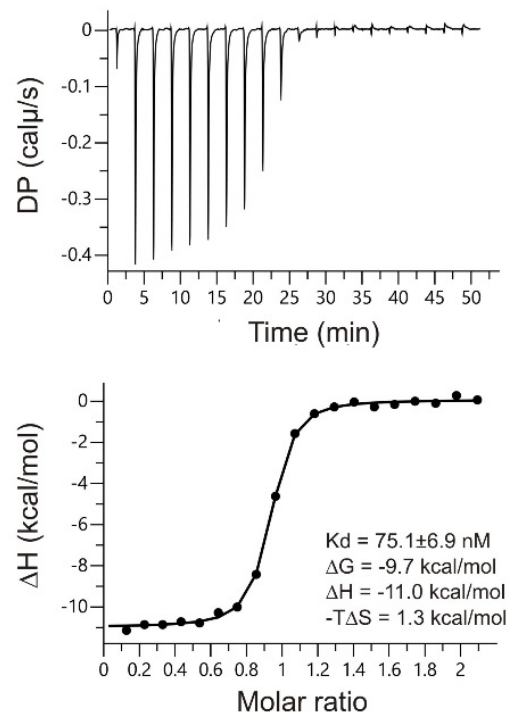

D
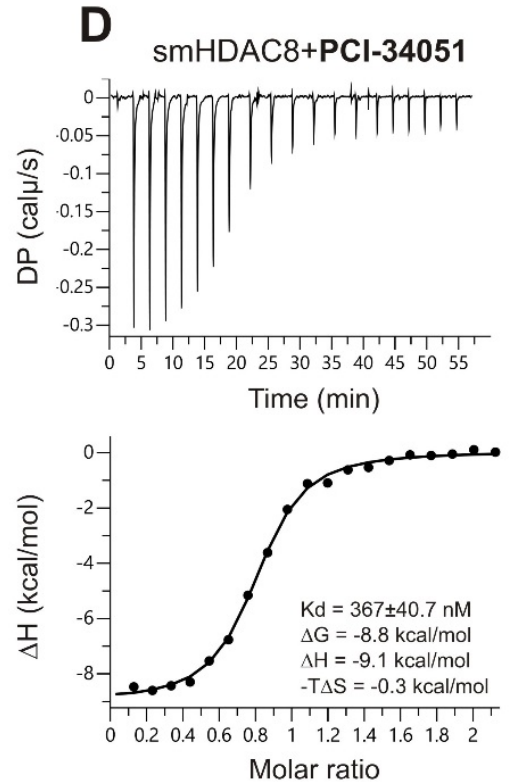

B hHDAC8+NCC-149
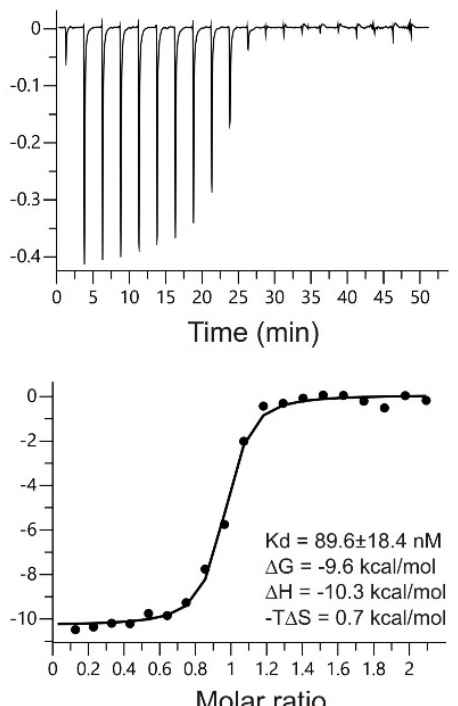

E
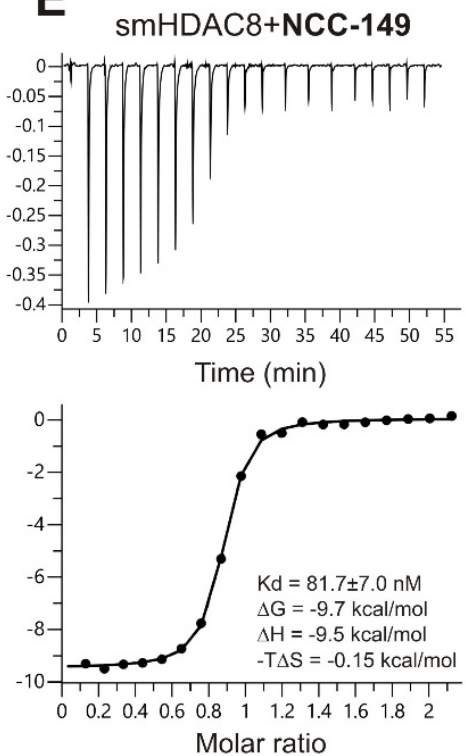

C

hHDAC8+QSN
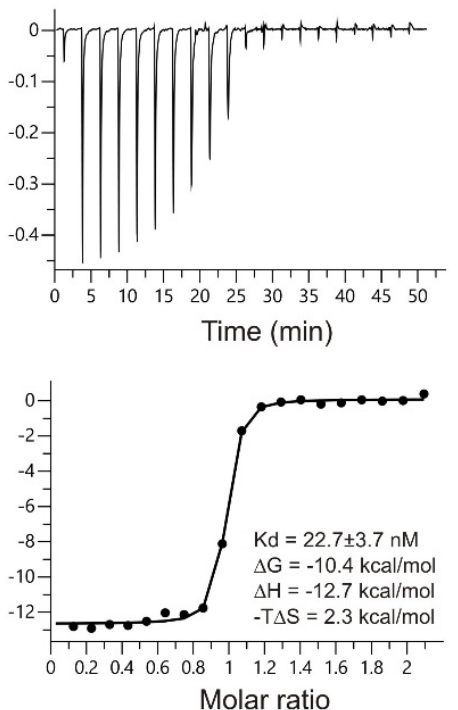

F
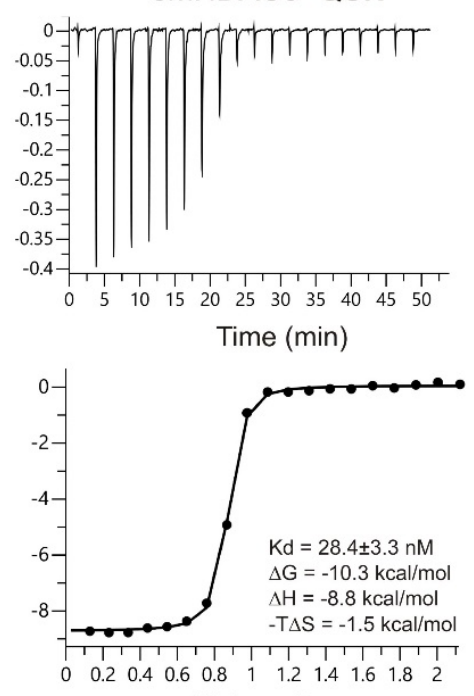

Molar ratio 


\section{Figure 3.}
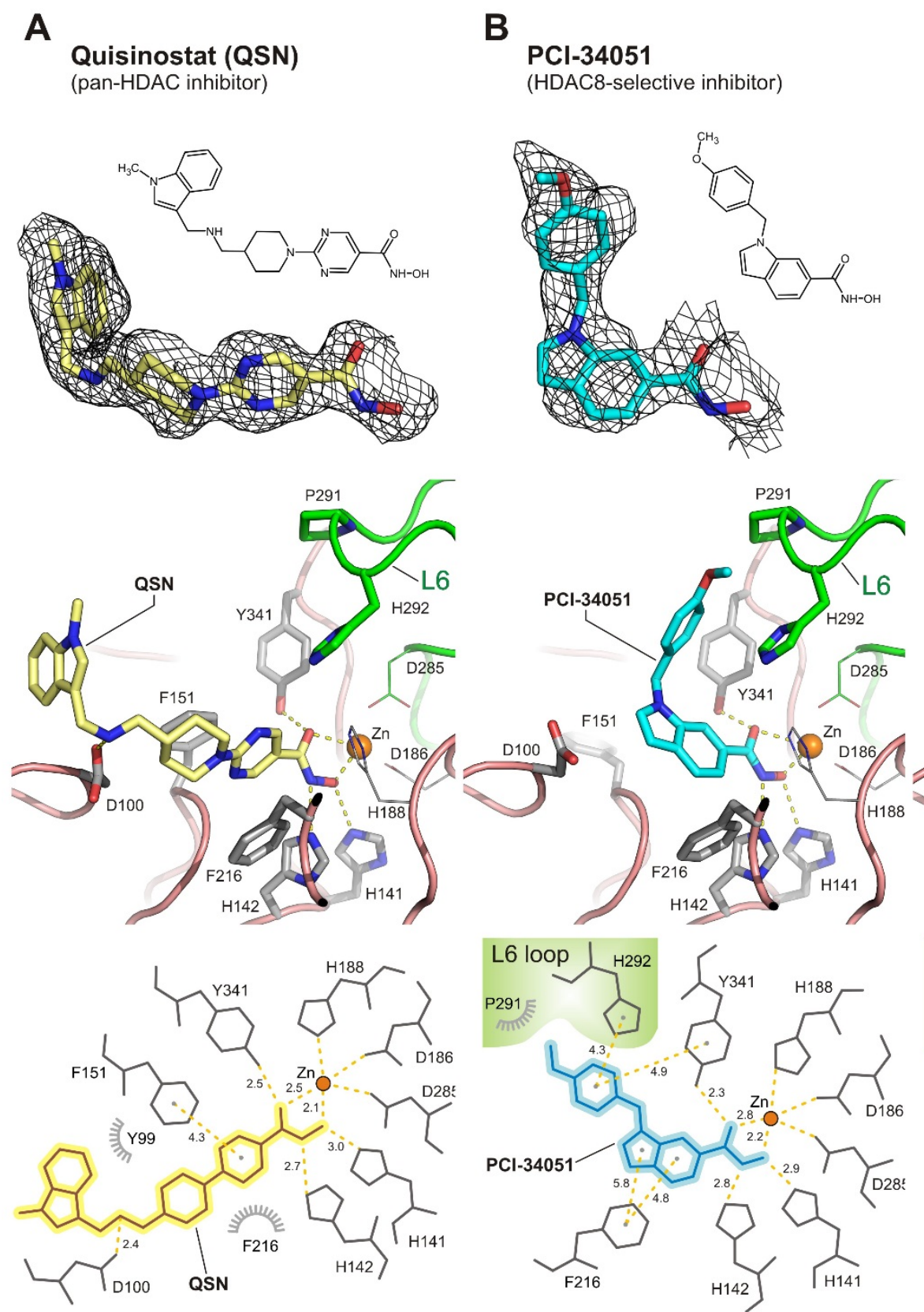

\section{B}

$\mathrm{PCl}-34051$

(HDAC8-selective inhibitor)
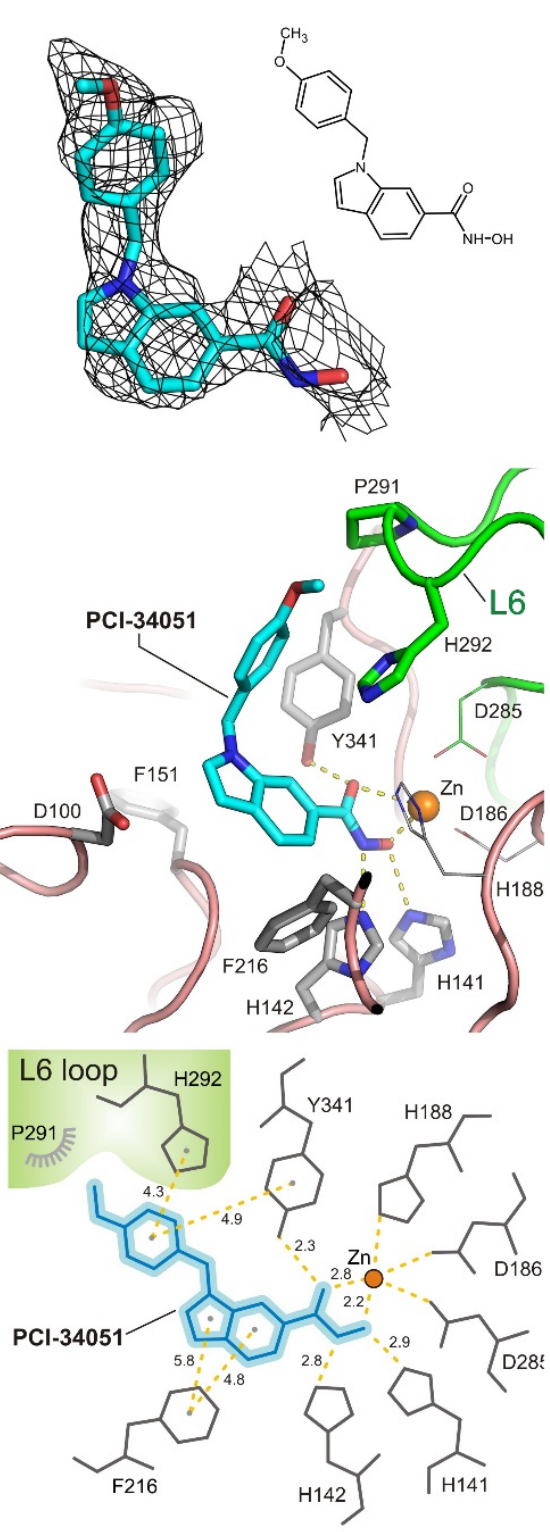

\section{C}

NCC-149

(HDAC8-selective inhibitor)
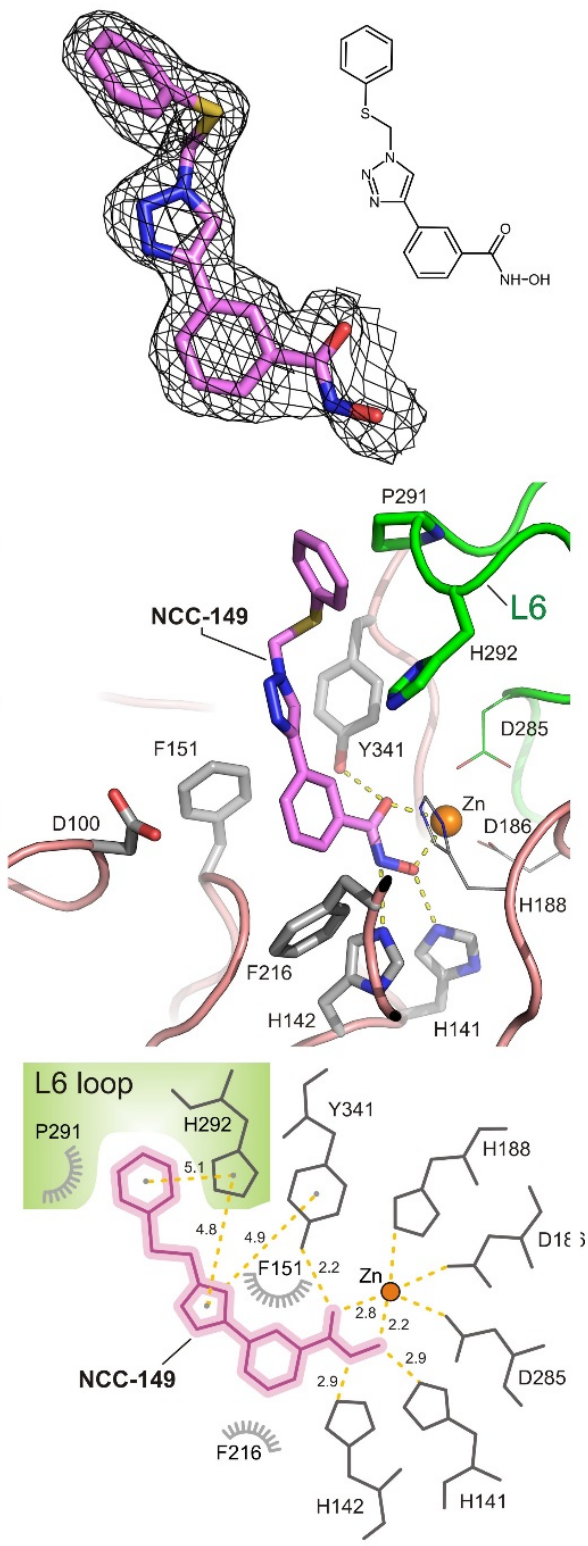
Figure 4.

A
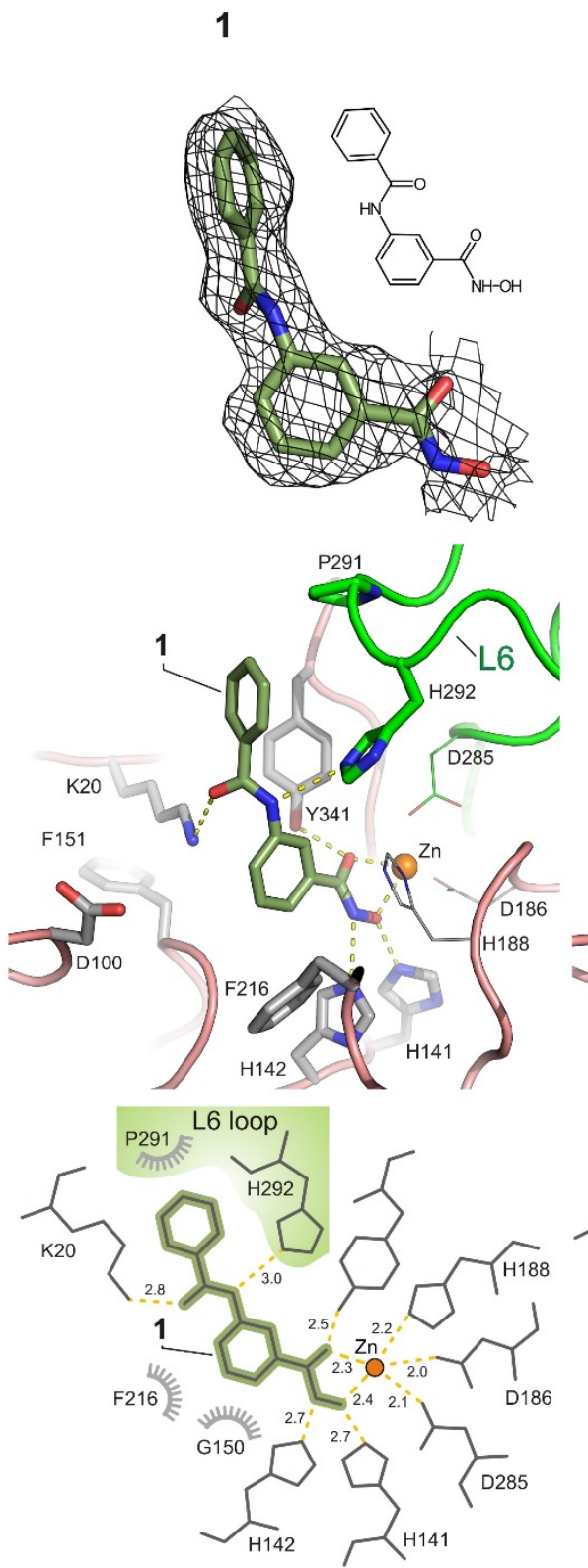

B
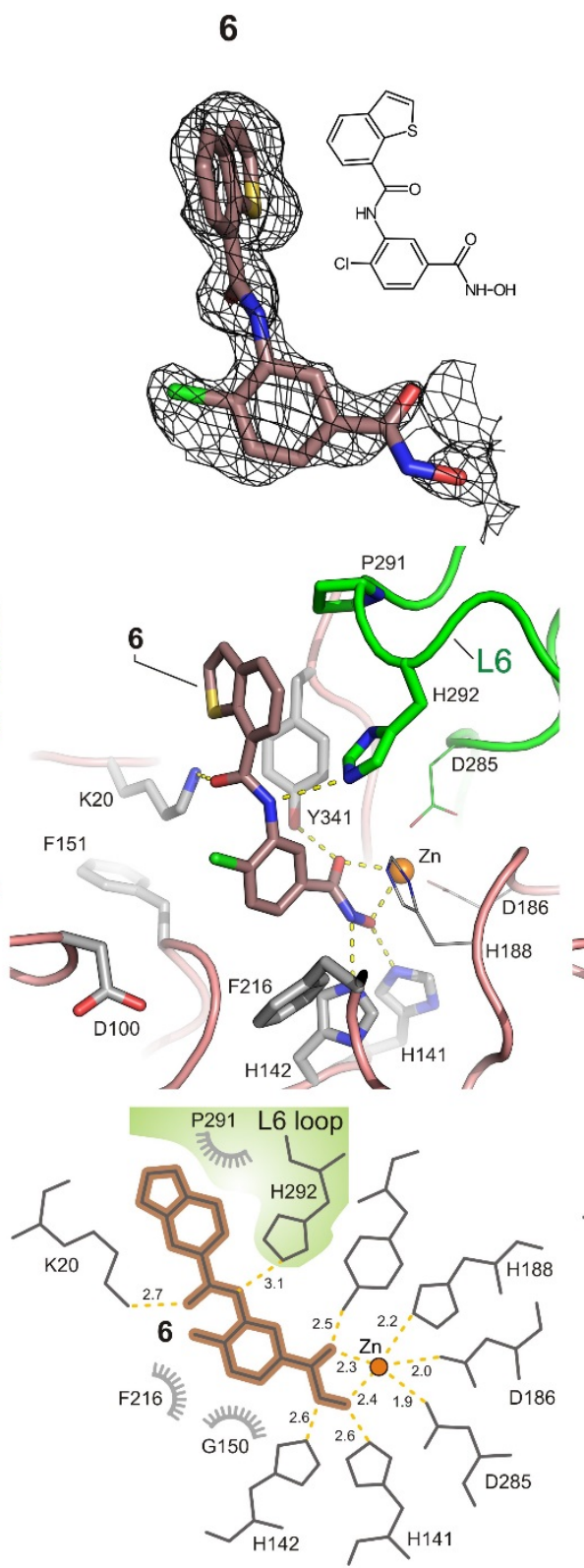

C
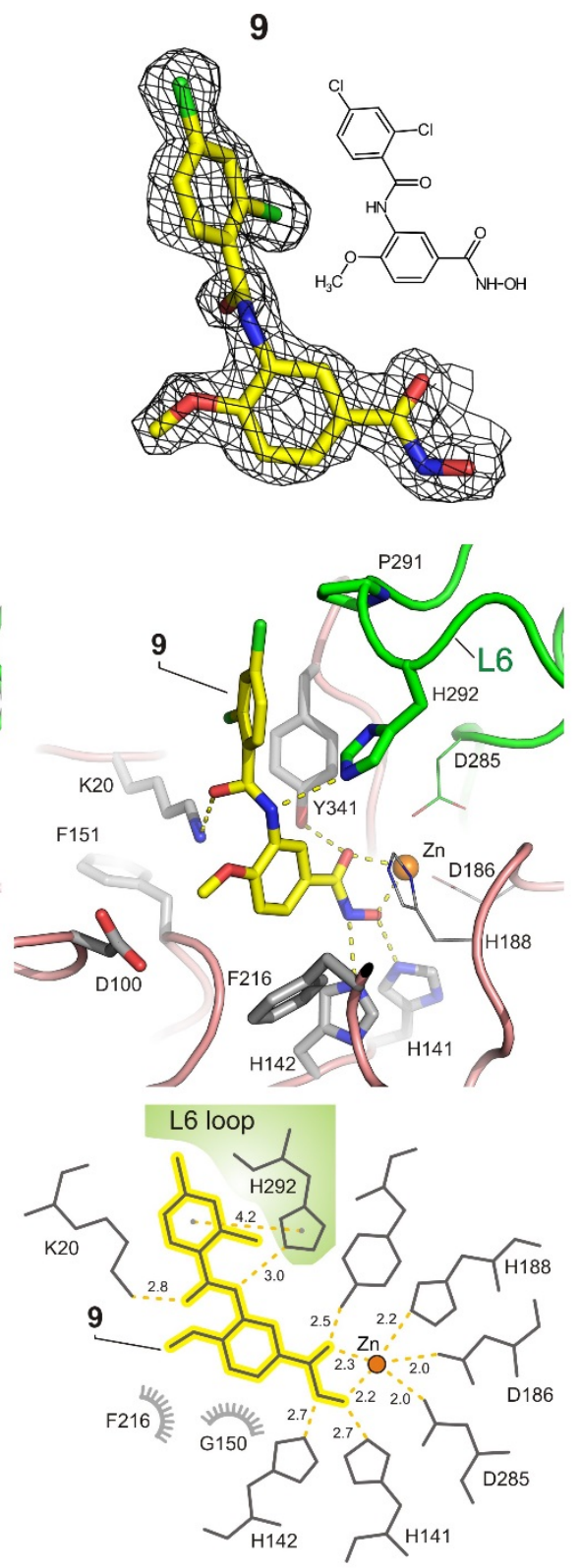


\section{Figure 5.}

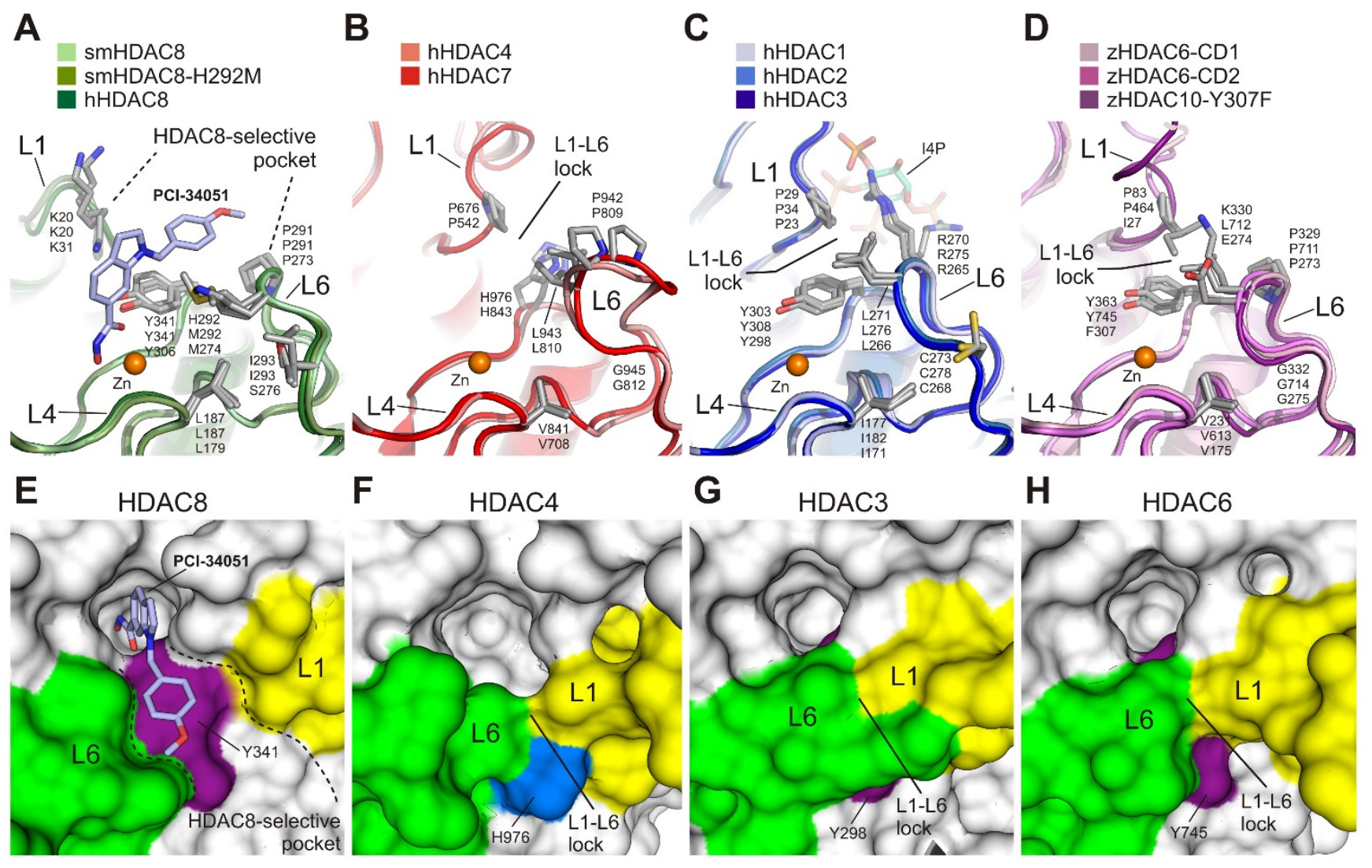




\section{Figure 6.}

A

smHDAC8 hHDAC8

hHDAC1

hHDAC2

hHDAC3

hHDAC4

hHDAC5

hHDAC7

hHDAC9

hHDAC6-CD1

hHDAC6-CD

hHDAC10

hHDAC11
L1 loop

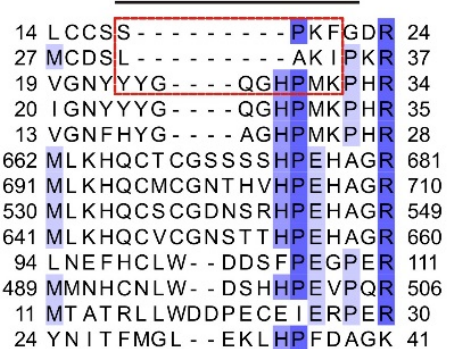

L4 loop

182 Y VDLDLHHGDGV 193 174 Y VDLDL HHGDGV 185 172 Y I D I D I HHGDGV 183 173 Y ID IDIHHGDGV 184 166 Y I D I D I HHGDGV 177 836 I VDWDVHHGNGT 847 866 I VDWD I HHGNGT 877 705 I VDWDVHHGNGT 716 816 I VDLDVHGNGT 827 827 249 I VDWDVHHGQGT 260 645 I VDWDVHHGNGT 656 168 VVDWDVHHGQG I 179

B

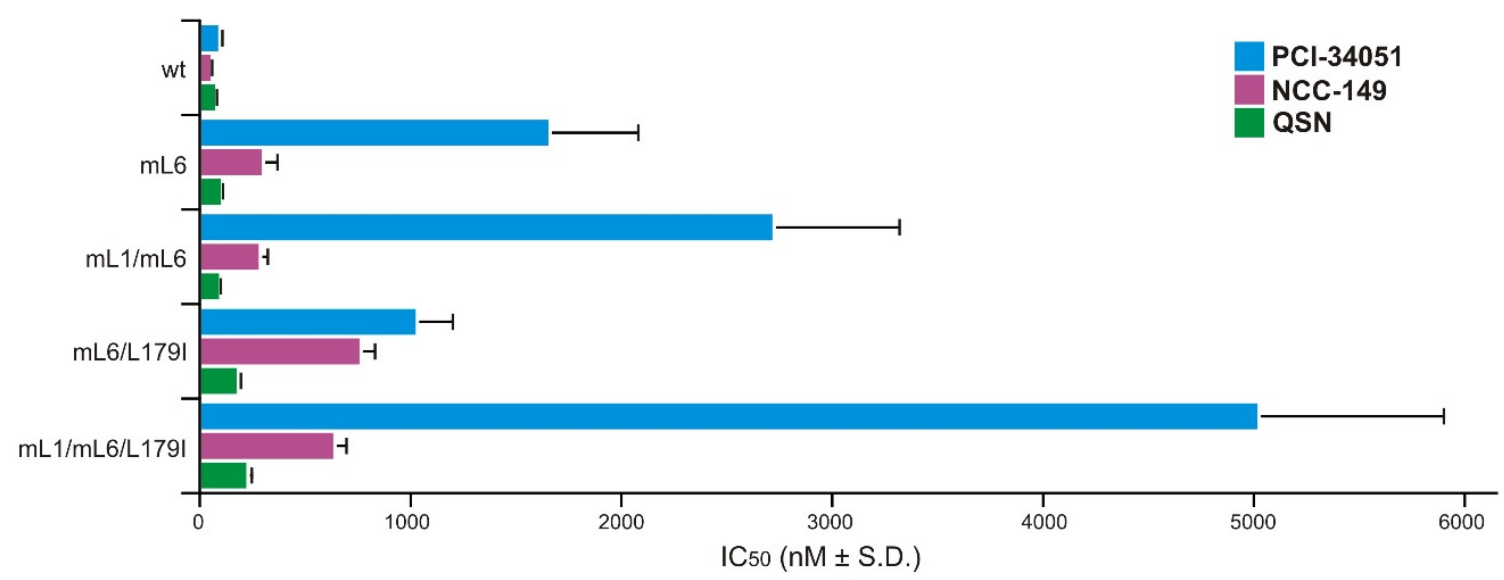

C

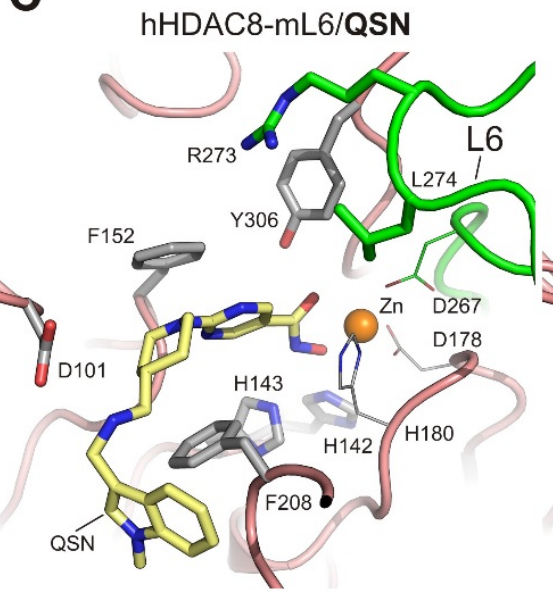

Superposition of hHDAC8-mL6/QSN and $\mathrm{hHDAC} 8 / \mathrm{PCl}-34051$
L6 loop

279 VVQCGAD CLAT - DPHR |FRLT (17) LSGYL 320 261 VLQLGADT I AG - - DPMCSFNMT PVG I G 285 258 VLQCGSDSLSG - DRLGCFNLT I KGHA 282 259 VLQCGADSLSG--DRLGCFNLT VKGHA 283 253 VLQCGADSLGC - DRLGCFNLS I RGHG 277 928 LVSSGFDAVEGHPTPLGGYNLS ARCFG 954 958 LVSAGFDAVEGHLSPLGGYSVT ARCFG 984 797 LVSAGFDAAEGHPAPLGGYHVS AKCFG 823 908 LVSAGFDALEGHTPPLGGYKVT AKCFG 934 340 LVAAGFDALQG - DPKGEMAAT PAGFA 364 736 LVSAGFDAARG - DPLGGCQVS PEGYA 760 259 LVSAGFDSA I - - DPEGQMQAT PECFA 283 255 VYNAGTDILEG - - DRLGGLS IS PAG IV 279 


\section{Table of Contents Graphic}

\section{Molecular basis of HDAC8 selective inhibition}
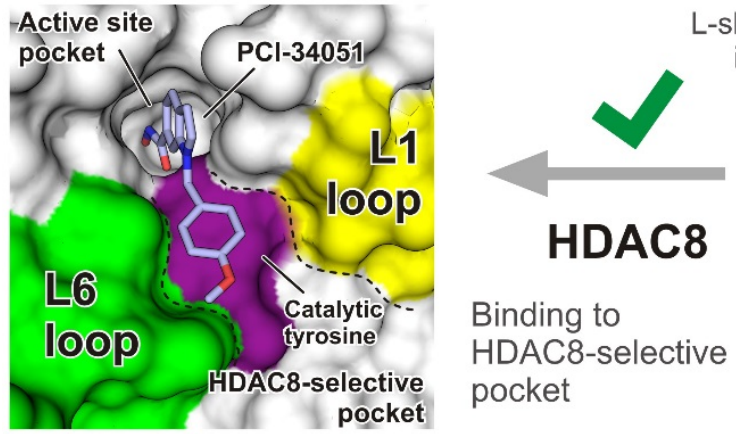

-shaped HDAC8-selective inhibitor (PCl-34051)
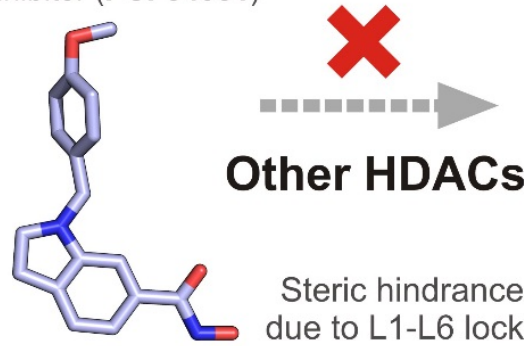

Steric hindrance due to L1-L6 lock

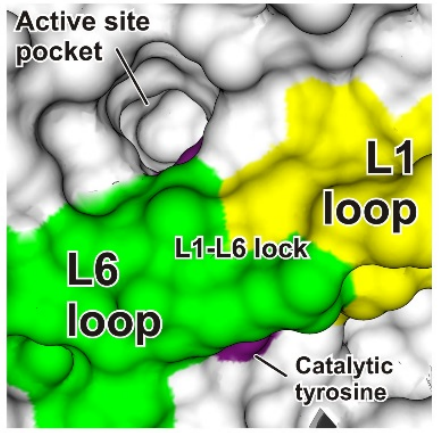

